\title{
Article
}

\section{Sox2 modulation increases naïve pluripotency plasticity}

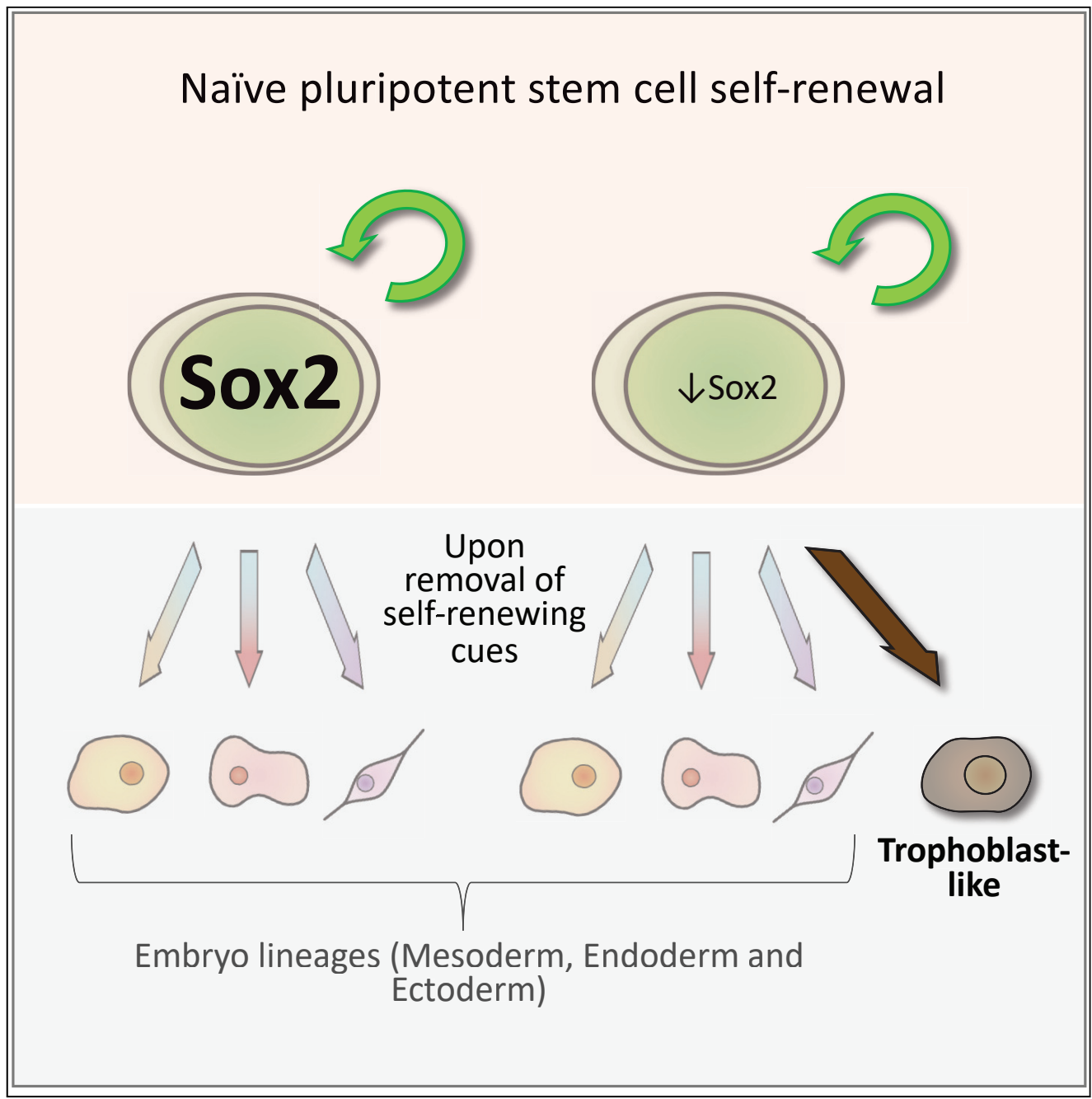

Kathryn C.

Tremble, Giuliano

G. Stirparo, Lawrence E. Bates, ..., BonKyoung Koo, Paul Bertone, José C.R. Silva

jose_silva@grmh-gdl.cn

HIGHLIGHTS

Low Sox2 expression is sufficient for naive pluripotent stem cell selfrenewal

Low Sox2 expression does not impair neurectoderm differentiation in vitro

Low Sox2 expression impairs Oct4 genomic occupancy

Low Sox2 increases naive pluripotent stem cell plasticity in vitro and in vivo 


\title{
Article \\ Sox2 modulation increases naïve pluripotency plasticity
}

\author{
Kathryn C. Tremble, ${ }^{1,2}$ Giuliano G. Stirparo, 1,7 Lawrence E. Bates, ${ }^{1,7}$ Katsiaryna Maskalenka, ${ }^{1,2}$ Hannah T. Stuart, \\ Kenneth Jones, ${ }^{1}$ Amanda Andersson-Rolf, ${ }^{1,3}$ Aliaksandra Radzisheuskaya, ${ }^{1,5}$ Bon-Kyoung Koo, 1,3,6 \\ Paul Bertone, ${ }^{4}$ and José C.R. Silva ${ }^{1,2,8, *}$
}

\section{SUMMARY}

Induced pluripotency provides a tool to explore mechanisms underlying establishment, maintenance, and differentiation of naive pluripotent stem cells (nPSCs). Here, we report that self-renewal of nPSCs requires minimal Sox 2 expression (Sox2-low). Sox2-low nPSCs do not show impaired neuroectoderm specification and differentiate efficiently in vitro into all embryonic germ lineages. Strikingly, upon the removal of self-renewing cues Sox2-low nPSCs differentiate into both embryonic and extraembryonic cell fates in vitro and in vivo. This differs from previous studies which only identified conditions that allowed cells to differentiate to one fate or the other. At the single-cell level self-renewing Sox2-low nPSCs exhibit a naive molecular signature. However, they display a nearer trophoblast identity than controls and decreased ability of Oct4 to bind naïve-associated regulatory sequences. In sum, this work defines wild-type levels of Sox2 as a restrictor of developmental potential and suggests perturbation of naive network as a mechanism to increase cell plasticity.

\section{INTRODUCTION}

The naive epiblast is pluripotent as it has the potential to differentiate into any cell type of the embryo proper but cannot form extraembryonic lineages. Naive pluripotent stem cells (nPSCs) can be captured in vitro from the epiblast in the form of embryonic stem cells (ESCs) (Evans and Kaufman, 1981; Martin, 1981) and through reprogramming of differentiated cells in the form of induced pluripotent stem cells (iPSCs) (Takahashi and Yamanaka, 2006). ESCs and iPSCs are therefore excellent model systems to study the molecular mechanisms underlying pluripotency. Recently it has been demonstrated that treatment of ESCs with specific small molecules induces expanded differentiation potential (Yang et al., 2017a, 2017b). These cells could contribute to both trophectoderm and inner cell mass (ICM) in blastocyst chimaeras. However, the mechanism underlying this has not been elucidated.

Sox2 is a member of the SRY-related HMG-box family of transcription factors (Wright et al., 1993) and is a core pluripotency factor. Sox 2 knockout in embryos results in peri-implantation lethality and its deletion in ESCs results in loss of self-renewal with the cells becoming trophoblast-like stem cells (Avilion et al., 2003; Masui et al., 2007). Sox2 was originally discovered as a putative DNA-binding partner of the central pluripotency factor Oct4 (Chew et al., 2005; Rodda et al., 2005; Yuan et al., 1995). However, self-renewing Sox2 ${ }^{-/-}$ ESCs were generated in the presence of constitutive Oct4 expression, suggesting that the main role of Sox2 is to maintain Oct4 expression (Masui et al., 2007). Additionally, overexpression of Sox2 results in differentiation of ESCs (Kopp et al., 2008). It has been hypothesized that Sox2 acts as a neurectoderm specifier, which needs to be in balance with mesendoderm specifiers to result in pluripotency (Loh and Lim, 2011; Thomson et al., 2011). Despite these studies, the role and requirement of Sox 2 in naive pluripotency remains unclear.

Here we utilized the process of induced pluripotency to investigate the biological role of Sox2. This uncovered the surprising role of Sox 2 in restricting the potency of nPSCs to embryonic lineages only. This impacts on both the understanding of the role of core naive pluripotency factors and on the molecular basis governing the potency of nPSCs.

${ }^{1}$ Wellcome Trust-Medical Research Council Cambridge Stem Cell Institute, University of Cambridge, Cambridge, UK

${ }^{2}$ Department of Biochemistry, University of Cambridge, Cambridge, UK ${ }^{3}$ Department of Genetics, University of Cambridge, Cambridge, UK

${ }^{4}$ Department of Medicine, Alpert Medical School, Brown University, Providence, Rl USA

${ }^{5}$ Present address: Cell Biology Program and Center for Epigenetics, Memorial Sloan Kettering Cancer Center, New York, USA ${ }^{6}$ Present address: MBA, Institute of Molecular Biotechnology of the Austrian Academy of Sciences, Vienna, Austria

${ }^{7}$ These authors contributed equally

${ }^{8}$ Lead contact

*Correspondence: jose_silva@grmh-gdl.cn https://doi.org/10.1016/j.isci. 2021.102153 
A

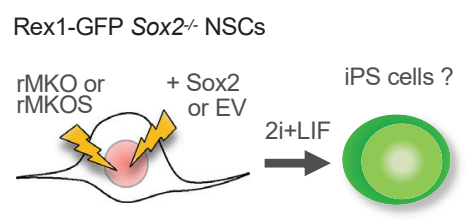

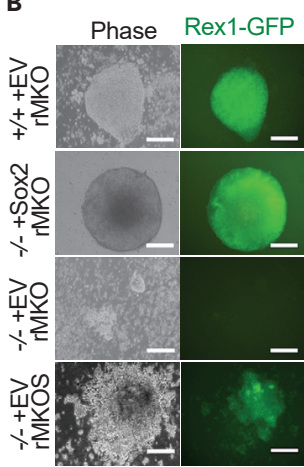

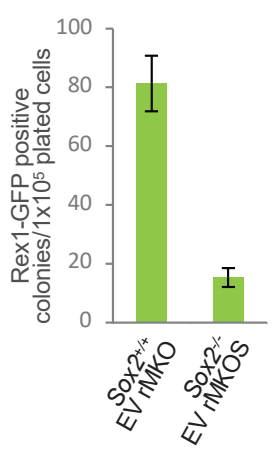

D

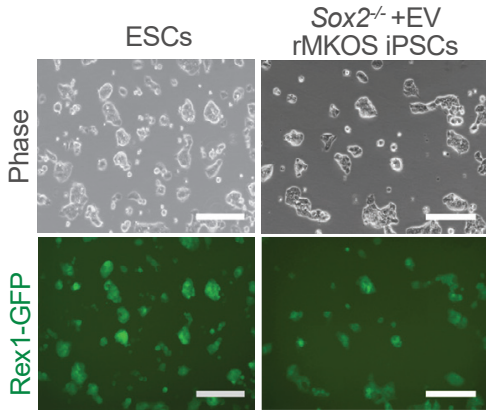

E

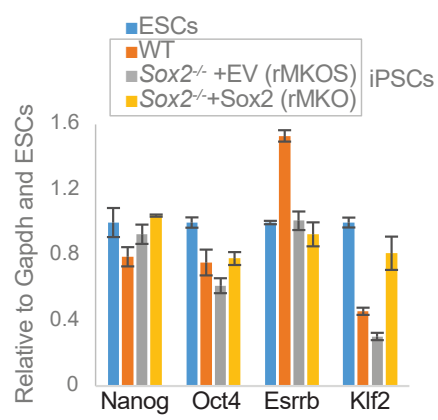

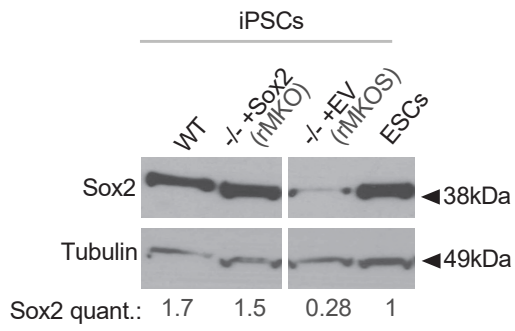

G

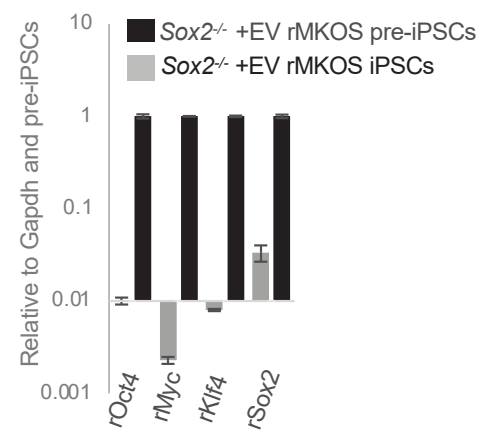

Figure 1. Generation of self-renewing iPSCs expressing low levels of Sox2

(A) Diagram of experimental design. rMKO and rMKOS indicate use of retroviral (r) vectors containing reprogramming cMyc (M), KIf4 (K), Oct4 (O), and Sox2 (S) transgenes. + EV (empty vector) or + Sox2 represent use of a PiggyBac plasmid containing a CAG promoter driving constitutive expression of either an empty or Sox2 transgene, respectively.

(B) Phase and GFP images of emerging Rex1-GFP + iPSC colonies $(n=3)$.

(C) Rex1-GFP + colony counts for indicated genotypes $(n=3)$.

(D) Phase and GFP images of Sox2 ${ }^{-1-}$ rMKOS iPSCs in 2iLIF post-selection. Rex1-GFP ESCs are shown as control.

(E) qRT-PCR analysis for indicated pluripotency associated factors in iPSC and control ESC lines.

(F) Western blot of Sox2 $(\approx 40 \mathrm{kDa})$ and Tubulin $(\approx 50 \mathrm{kDa})$ in iPSC and control ESC lines in 2iLIF with Sox2 quantification relative to ESCs, normalized to tubulin. Gap in Western blot represents removal of a non-relevant lane and image corresponds to same film exposure.

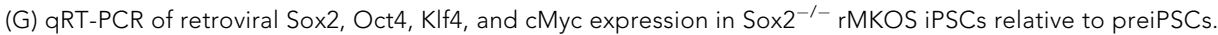

Scale bars $=200 \mu \mathrm{m}$.

Error bars indicate standard deviation of replicate GPCR reactions $(n=3)$.

\section{RESULTS}

\section{Low Sox 2 expression is compatible with nPSC self-renewal}

To investigate the role of Sox 2 in the process of induced pluripotency, we used neural stem cells (NSCs) as a source of somatic donor material. To attempt the generation of Sox $2^{-1-}$ iPSCs, we made initially Sox $2^{-1-}$ NSCs using CRISPR/Cas9 (Figures S1A-S1D). Clonal lines were confirmed to have a frameshift deletion in the Sox2 codon, resulting in loss of Sox2 protein (Figures S1A and S1B). The Sox2 ${ }^{-1-}$ NSCs maintained NSC morphology, proliferative ability, and expression of NSC markers (Figures S1C and S1D). The Sox $2^{-/-}$NSCs contained GFP and the blasticidin resistance genes under the endogenous Rex1 regulatory sequences to allow identification and selection for nPSC identity (Wray et al., 2011). Sox $2^{-1-}$ NSCs were induced to reprogram by combining four or three of the classic Yamanaka retroviral factors: cMyc, Klf4, Oct4 and Sox2 (rMKOS) or cMyc, KIf4, and Oct4 (rMKO) (Takahashi and Yamanaka, 2006) (Figure 1A). Retroviral promoters are active in somatic cells but become epigenetically silenced in naive pluripotency (Hotta and Ellis, 2008). Therefore, we hypothesized that the retroviral Sox2 would drive reprogramming but become silenced after stabilization of the network. We also used defined culture conditions containing inhibitors of Mek/Erk and Gsk3b signaling (2i) supplemented with Leukaemia inhibitory factor (LIF) for optimal reprogramming efficiency (Silva et al., 2008). Sox $2^{-/-}$NSCs were able to 
A

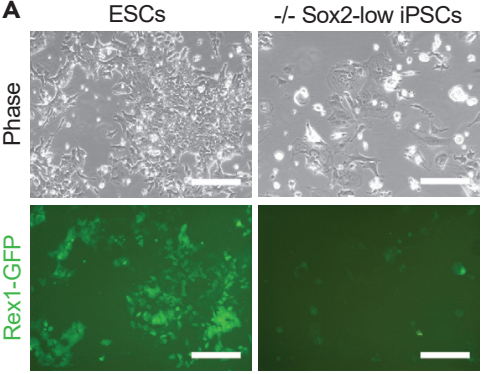

B

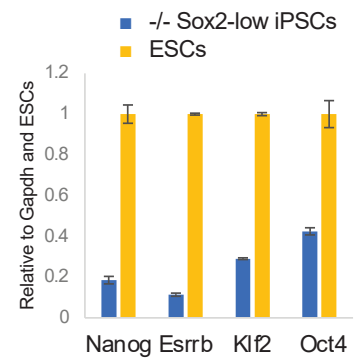

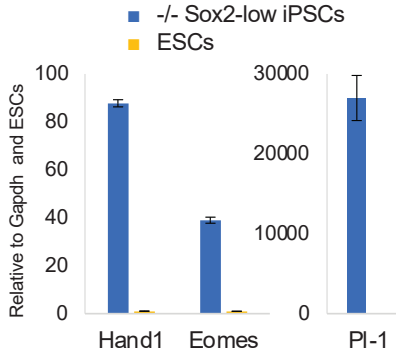

D

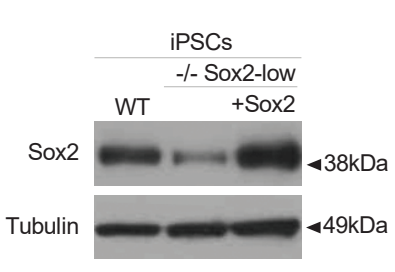

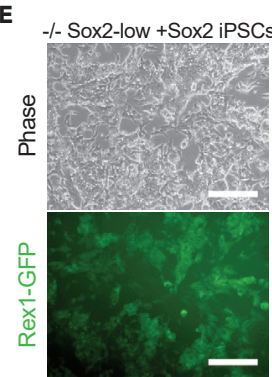

$\mathbf{F}$

$-1-$ Sox2-low iPSCs

- --Sox2-low +Sox2 iPSCs - ESCs

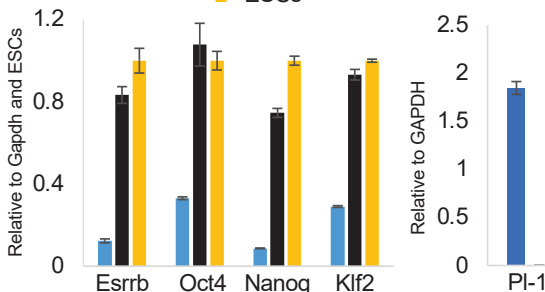

Figure 2. Sox2-low iPSCs do not self-renew in serum plus LIF

(A) Phase and Rex1-GFP images of Sox2 $2^{-1-}$ rMKOS (-/-Sox2-low) iPSCs and ESCs at passage 0 after medium switch from 2i plus LIF into serum plus LIF.

(B and C) qRT-PCR analysis of pluripotency markers (B) and trophoblast markers (C) 3 days after switching into serum plus LIF medium.

(D) Western blot of Sox2-low iPSCs in 2i plus LIF with and without rescue Sox2 transgene (+Sox2). Rex1-GFP WT iPSCs were used as control for Sox2 protein levels.

(E) Phase and GFP images of -/- Sox2-low + Sox2 rescue iPSCs after 5 passages in serum plus LIF.

(F) qRT-PCR analysis, after 3 days in serum plus LIF, of pluripotency factors and PI-1 in -/-Sox2-low iPSCs with and without a rescue Sox2 transgene. ESCs were provided as control in the qRT-PCR analysis of pluripotency factors.

Scale bars $=200 \mu \mathrm{m}$. Error bars indicate standard deviation of replicate qPCR reactions $(n=3)$.

reprogram upon addition of a constitutive exogenous Sox2 transgene (Figure 1B). In the absence of retroviral Sox2, Sox2 ${ }^{-/}$NSCs failed to upregulate the Rex1-GFP reporter (Figure 1B). Surprisingly, multiple Sox $2^{-/}$Rex1-GFP + colony-like groups of cells emerged when using rMKOS (Figure 1B). Independent experiments showed this to be 5 times less efficient compared to the reprogramming of WT NSCs (Figure 1C). Reprogrammed Sox2 ${ }^{-1-}$ rMKOS iPSCs were passagable in 2iLIF culture conditions and expressed naive pluripotency markers (Figures 1D and 1E). Surprisingly, they also expressed Sox2 protein at very low levels (Figure 1F), which was due to a failure to fully silence retroviral Sox2 (Figure 1G). Importantly, this data indicates a strong selective pressure for cells expressing a minimal level of Sox 2 protein and suggests that low Sox2 expression is compatible with maintenance of a nPSC molecular identity. Hereafter, we will refer to these Sox $2^{-1-}$ rMKOS iPSCs as Sox2-low iPSCs for simplicity.

\section{Sox2-low iPSCs differentiate in serum plus LIF self-renewing conditions}

It has previously been reported that Sox2 repression in ESCs results in loss of pluripotency and differentiation toward the trophoblast lineage in serum plus LIF (SLIF) conditions (Masui et al., 2007). Therefore, we attempted to culture Sox2-low iPSCs in SLIF. Strikingly, Sox2-low iPSCs downregulated the Rex1-GFP reporter, and some cells gained a trophoblast-like morphology (Figure 2A). They also downregulated pluripotency marker expression and upregulated trophoblast markers (Figures 2B and 2C). In addition, cells were not passageable, demonstrating loss of self-renewal. To ensure that the differentiation phenotype was due to low Sox2 expression, we generated Sox2-low rescue iPSCs by transfecting these with a constitutive Sox2 transgene in 2iLIF (Figure 2D). Upon SLIF medium switch, and in contrast to Sox2-low iPSCs, rescue iPSCs self-renewed, maintained naive pluripotent gene expression and did not upregulate trophoblast marker expression (Figures 2E and 2F). 
A
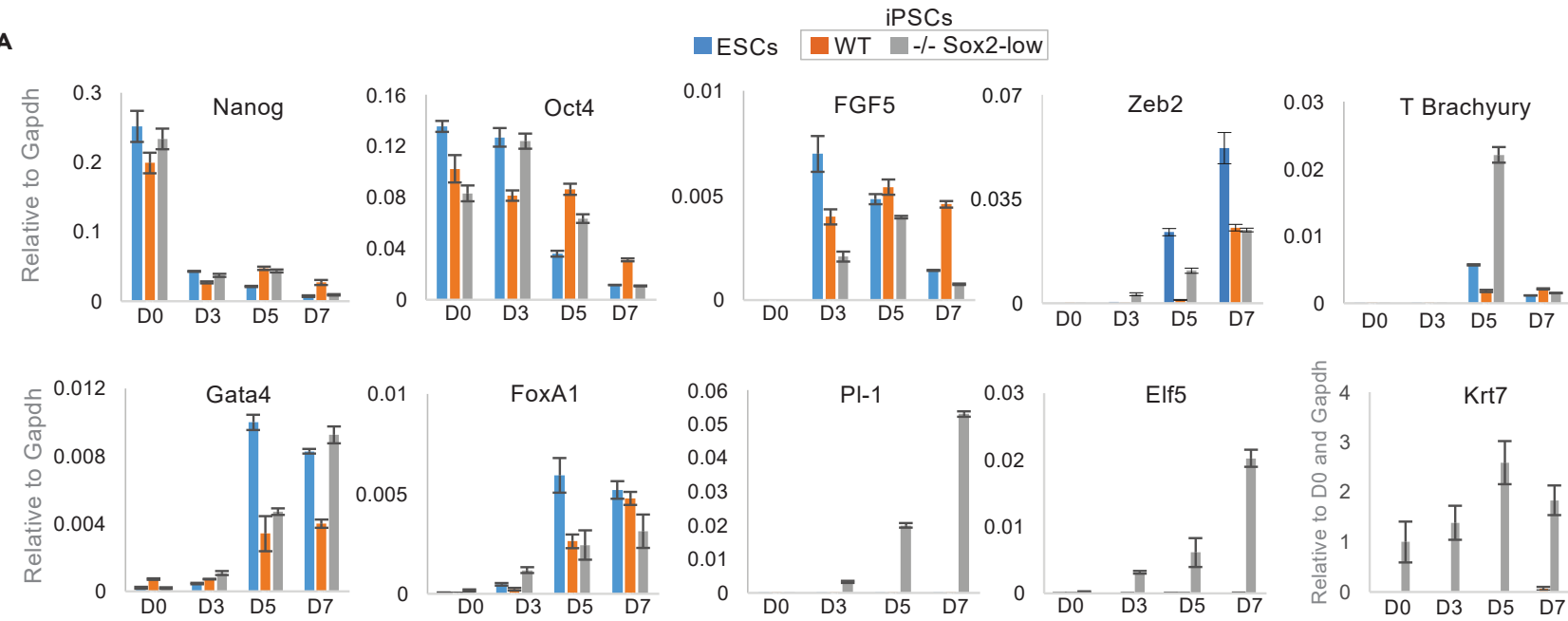

B

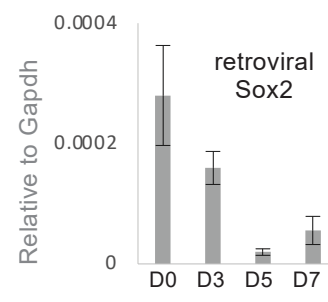

C

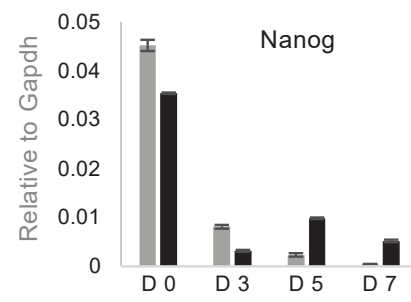

-/- Sox2-low

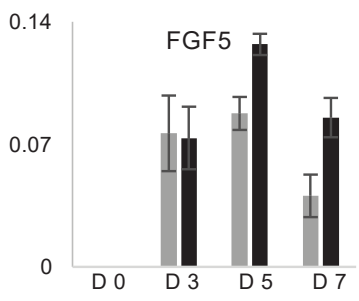

PI-1

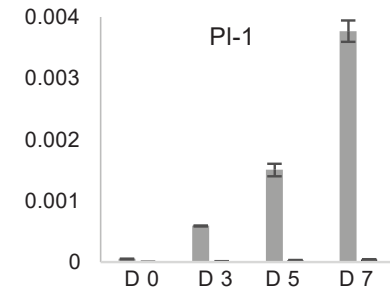

D

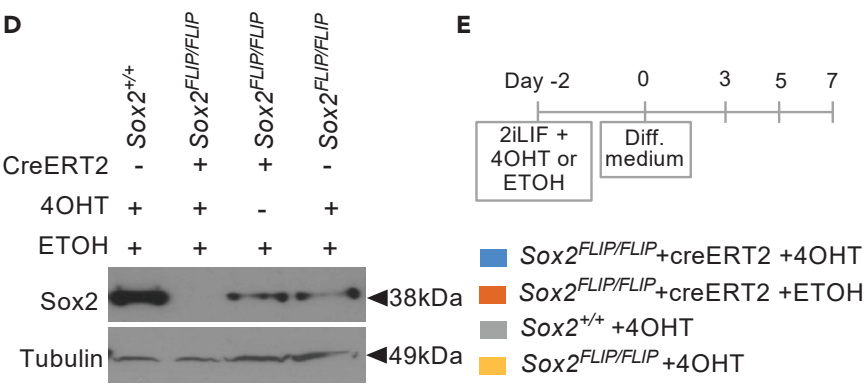

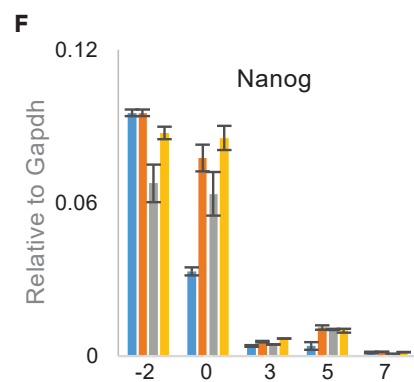

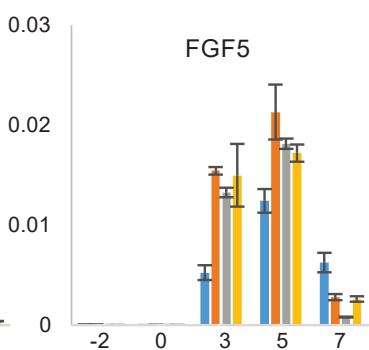

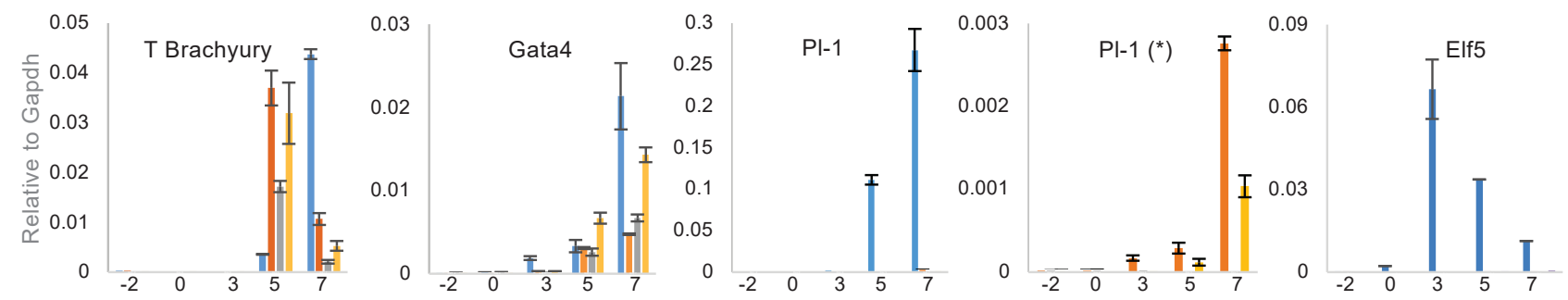


Figure 3. Sox2-low and null nPSCs differentiate into extraembryonic and all embryonic lineages

(A) qRT-PCR analysis of embryoid body assay using Sox2 $2^{-1-}(-/-)$ Sox2-low iPSCs, control iPSCs (WT) and ESCs showing RNA expression of markers of pluripotency (Nanog, Oct4), ectoderm (FGF5), mesoderm (T Brachyury and Zeb2), endoderm (FoxA1 and Gatat4) and trophoblast (PI-1 and Elf5).

(B) qRT-PCR analysis of embryoid body assay for retroviral Sox2 expression in -/- Sox2-low iPSCs.

(C) qRT-PCR analysis of embryoid body assays of -/ - Sox2-low and rescue (+Sox2) iPSCs, for expression of pluripotency (Nanog), late epiblast (FGF5) and trophectoderm (PL-1) markers.

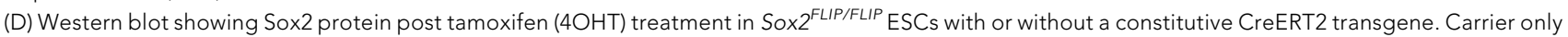
(ethanol, ETOH) and Sox $2^{+/+}$ESCs were used as additional controls.

(E) Experimental design of embryoid body assay and of Sox2 deletion in Sox2 FLIP/FLIP ESCs.

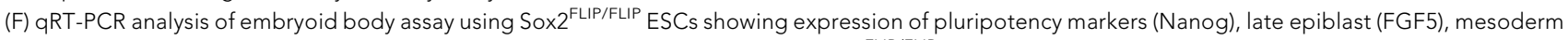

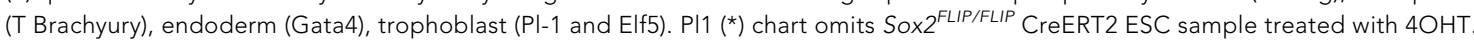

Error bars indicate standard deviation of replicate qPCR reactions $(n=3)$.

These results suggest that under weaker self-renewing culture conditions low levels of Sox 2 are not sufficient to sustain a naive pluripotent identity. It also showed that at least a proportion of the cells differentiate toward the trophoblast lineage.

\section{Reduced Sox 2 expression is associated with increased plasticity in vitro}

To functionally characterize the potency of Sox2-low iPSCs we investigated their differentiation capacity in embryoid body (EB) assays. These efficiently downregulated pluripotency genes and upregulated both endoderm and mesoderm differentiation markers. Importantly, retroviral Sox2 was not upregulated during differentiation (Figure 3B). Because of the observed upregulation of trophoblast markers in Sox2-low iPSCs in SLIF, we also explored the expression of these. In contrast to control lines, Sox2-low iPSCs upregulated expression of trophoblast markers during EB differentiation (Figure 3A). Interestingly, and in contrast to Elf5 and Cdx2, the trophoblast marker Krt7 was already expressed prior to cell differentiation. This phenotype was fully rescuable upon the restoration of Sox 2 protein levels by the transfection of a constitutive Sox2 transgene (Figures 2D and 3C). These results suggest that Sox2-low iPSCs have increased plasticity as they can differentiate into both embryonic and extraembryonic lineages.

Next we went on to test our findings in an independent nPSC system (Figures 3D-3F and S2A-S2C). Sox2 FLIP/FLIP ESCs have previously been generated, which delete endogenous Sox2 expression upon exposure to Cre (Fig-

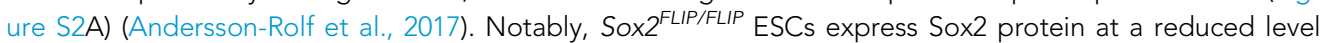
compared to WT ESCs (Figure 3D). This is likely the result of the inserted cassette sequences interfering with

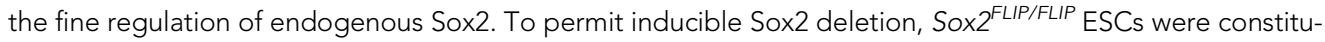
tively transfected with creERT2. These were then subjected to a tamoxifen time course to establish the earliest time point at which Sox 2 protein is absent. By 36 hr Sox 2 protein was undetectable, whereas Nanog and Oct4 were still expressed at high levels at $48 \mathrm{hr}$, suggesting that differentiation had not occurred (Figures S2B and S2C). Therefore, to investigate the potency of Sox2-null ESCs, we treated CreERT2 Sox2 FLIP/FLIP ESCs with tamoxifen (4OHT) for $48 \mathrm{hr}$ and then performed EB differentiation (Figures 3D-3F). The absence of Sox 2 protein did not affect the expected kinetics of downregulation of pluripotency markers or the upregulation of differentiation markers of all 3 germ layers (Figure 3E). Importantly, absence of Sox2 expression was also associated with

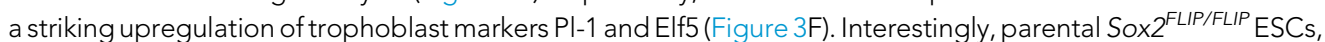
which exhibit lower Sox2 protein expression compared to control ESCs, also exhibit significant upregulation of the trophoblast marker PI-1 compared to control ESCs (Figure 3F).

Importantly, these results confirm that reduced Sox2 expression in nPSCs (iPSCs and ESCs) is associated with a gain in cell plasticity, that is, an ability to differentiate toward the extraembryonic trophoblast lineage in addition to the embryonic lineages.

\section{Low Sox 2 expression does not impair neurectoderm differentiation}

Sox 2 is thought to be required to drive ectoderm differentiation in pluripotent cells (Thomson et al., 2011). To examine the neurectoderm differentiation potential of these cells, we performed a neural monolayer differentiation (Ying et al., 2003). The Sox2-low iPSCs upregulated neural markers Sox1, Pax6, and Ascl1 to a similar level to WT iPSCs and Sox2-low rescue iPSCs and gained the characteristic neural rosette morphology, demonstrating efficient neural differentiation (Figures $4 \mathrm{~A}$ and $4 \mathrm{C}$ ). This also occurred without an increase of retroviral Sox2 expression (Figure 4B). These results suggest that reduced Sox2 expression does not impair robust neural differentiation in vitro. 
A

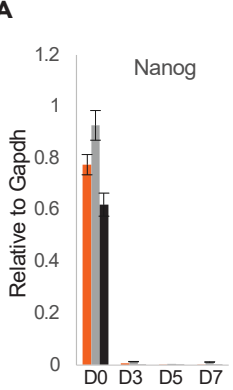

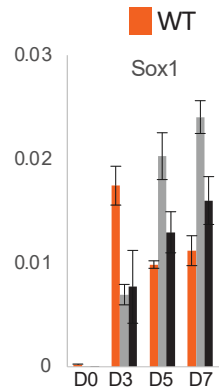

WT

-/- Sox2-low

0.02
- - Sox2-low +Sox2

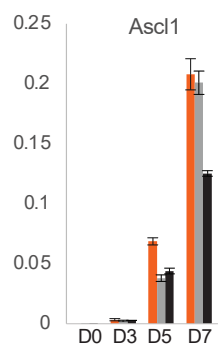

B

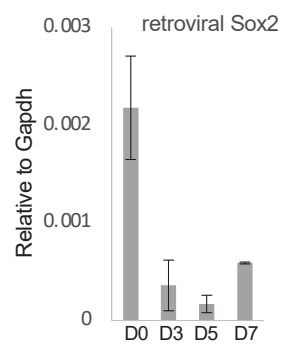

C Day 7 monolayer neuroectoderm differentiation

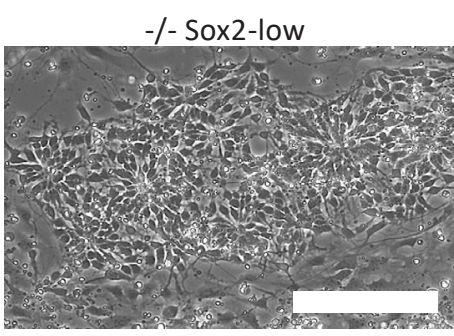
-/- Sox2-low +Sox2

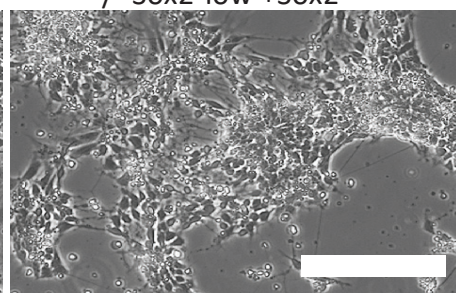

Figure 4. Low Sox2 expression is compatible with robust neuroectoderm differentiation (A) qRT-PCR analysis of Sox2 ${ }^{-/-}(-/-)$Sox2-low, -/- Sox2-low rescue (+Sox2) and WT iPSCs in a neurectoderm monolayer differentiation assay for markers of pluripotency (Nanog) and neurectoderm (Pax6, Ascl1, and Sox1). (B) gRT-PCR analysis of retroviral Sox2 in -1- Sox2-low iPSCs during neurectoderm monolayer differentiation. (C) Phase images of -/- Sox2-low iPSCs and Sox2-low rescue iPSCs on day 7 of the neurectoderm monolayer differentiation assay.

Error bars indicate standard deviation of replicate qPCR reactions $(n=3)$.

\section{Reduced Sox2 expression is associated with increased plasticity in vivo}

To investigate the effect of reduced Sox2 expression in differentiation in vivo, we performed morula aggregations with Sox2-low iPSCs. To visualize embryo chimerism we constitutively transfected Sox2-low iPSCs with MST-dsRed fluorescent protein. Contribution of Sox2-low iPS-derived cells could be seen in both epiblast and presumptive extraembryonic compartments at E7.5 (Figure 5A). In order to determine extraembryonic chimerism, we also generated Sox2-low and control iPSCs expressing constitutive GFP. As expected, WT iPSCs contributed exclusively to the E6.5 epiblast (Figures 5B-5D). In contrast, Sox2-low iPS-derived cells were found in both the epiblast and trophoblast, as defined by the AP2 $\gamma$ protein domain, compartments of the embryos (Figures 5B, 5C, 5E, and S3A). Lineage marker staining showed that $83 \%$ of Sox2-low chimaeras exhibit contribution to both the trophoblast and epiblast compartments (Figure 5D). In fact, $90 \%$ of all Sox2-low chimeras showed trophoblast contribution. Interestingly, some Sox2-low iPSderived cells exhibited co-expression of trophoblast marker AP-2 $\gamma$ and epiblast marker Oct4 (filled arrowheads, Figure 5C) and this occurred in both the epiblast and trophoblast embryo compartments suggesting that this is an intermediate stage of cells changing from a pluripotent epiblast into a trophoblast identity.

To define when Sox2-low iPS-derived cells start contributing to the trophoblast lineage, we injected these at the morula stage and assessed chimerism at the blastocyst stage. Interestingly, almost all of the chimaeras showed contribution to the epiblast only (Figures S3B and S3C). This data support in vitro assays showing that Sox2-low iPSCs only acquire trophoblast lineage differentiation potential when in an environment no longer supportive of naive pluripotent cell identity.

Overall, these data demonstrate that Sox2-low iPSCs are competent to contribute to both embryonic and extraembryonic embryo development. 


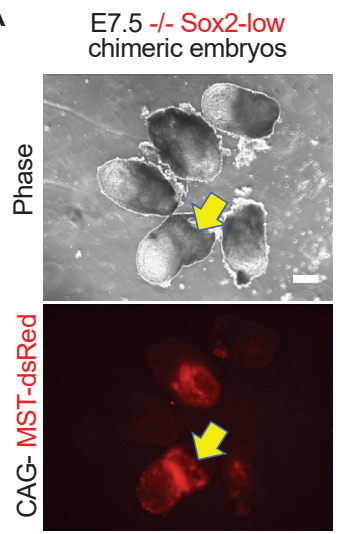

B

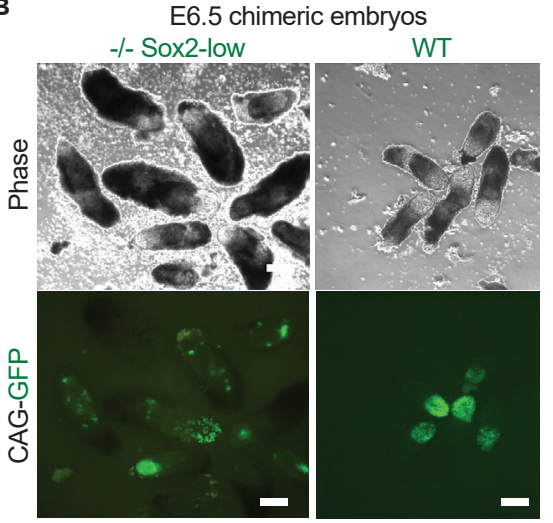

C
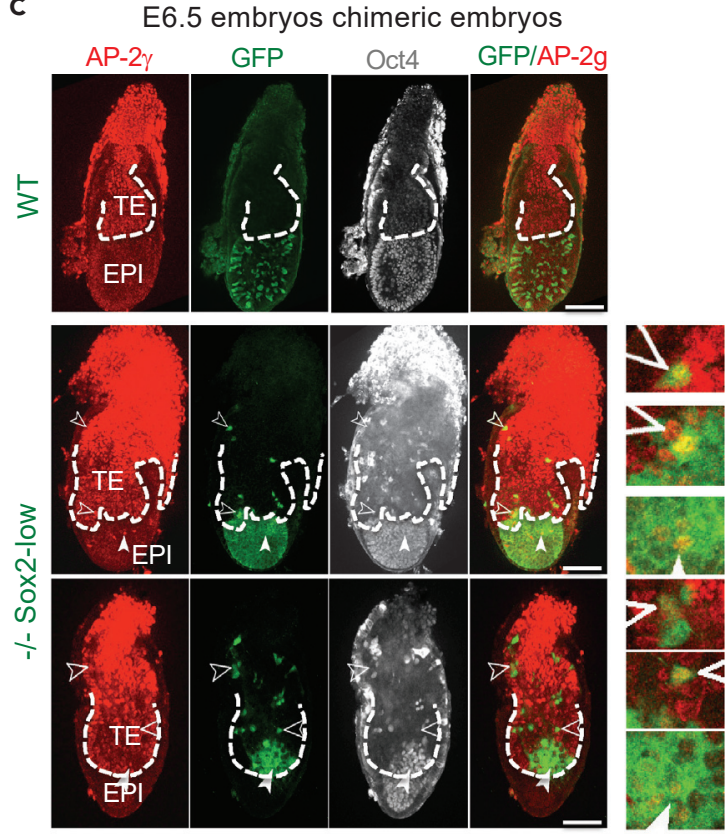

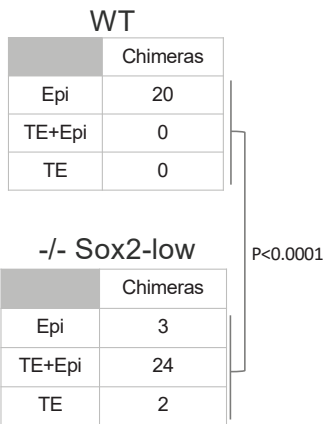

Figure 5. Sox2-low iPSCs exhibit increased plasticity in vivo

(A) Phase and red fluorescence images of E7.5 chimaeras of Sox2 ${ }^{-1-}$ (-/-) Sox2-low iPSCs expressing constitutively MST$\mathrm{dsRed}$ (red fluorescence). Arrow indicates presumptive contribution to extraembryonic lineage(s). A non-chimeric embryo is included above the chimeric embryo with the yellow arrow (toward the left) to act as a negative control. Scale bars = $200 \mu \mathrm{m}$.

(B) Phase and GFP images of E6.5 chimeric embryos generated with either - /- Sox2-low or WT iPSCs constitutively expressing a GFP transgene. Please note that apparent difference in size is due to -/-Sox2 chimeric embryos having been imaged with some maternal tissue still attached. Scale bars $=200 \mu \mathrm{m}$.

(C) Single confocal microscopy sections of indicated genotype chimeric E6.5 embryos stained with trophoblast (AP-2 $\gamma$ ) and epiblast (Oct4) markers. Filled arrowheads indicate examples of chimeric cells co-expressing Oct4 and AP-2 $\gamma$ markers. Non-filled arrowheads indicate examples of chimeric cells expressing AP-2 $\gamma$ only. Epiblast (EPI) and Trophoblast/trophectoderm (TE) embryo domains are separated by dashed line. Scale bars $=100 \mu \mathrm{m}$.

(D) Table showing compartmental contribution of WT or -/- Sox2-low iPSCs constitutively expressing a GFP transgene. Values in tables represent number of embryos. Fisher's exact test statistical analysis was used to calculate the significance of the difference in the proportion of embryos exhibiting trophectoderm contribution in the two groups. This was performed using GraphPad Prism software.

\section{Sox2-Iow iPSCs have weaker epiblast identity}

To ascertain the identity of Sox2-low iPSCs we performed single-cell RNA-seq on these and control WT and Sox2-low + Sox2 rescue iPSCs. Principle component analysis showed that Sox2-low iPSCs can be separated 
A

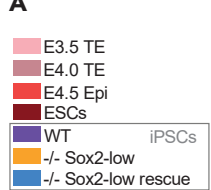

Dendrogram (log2FPKM > 0 - agglomeration method ward.D2)

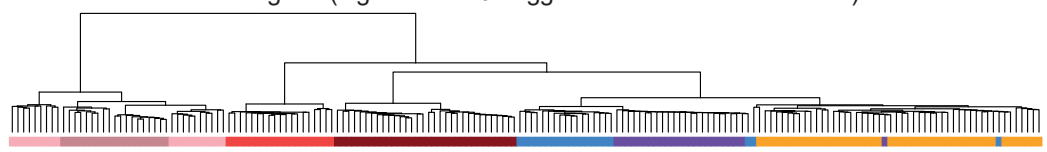

B
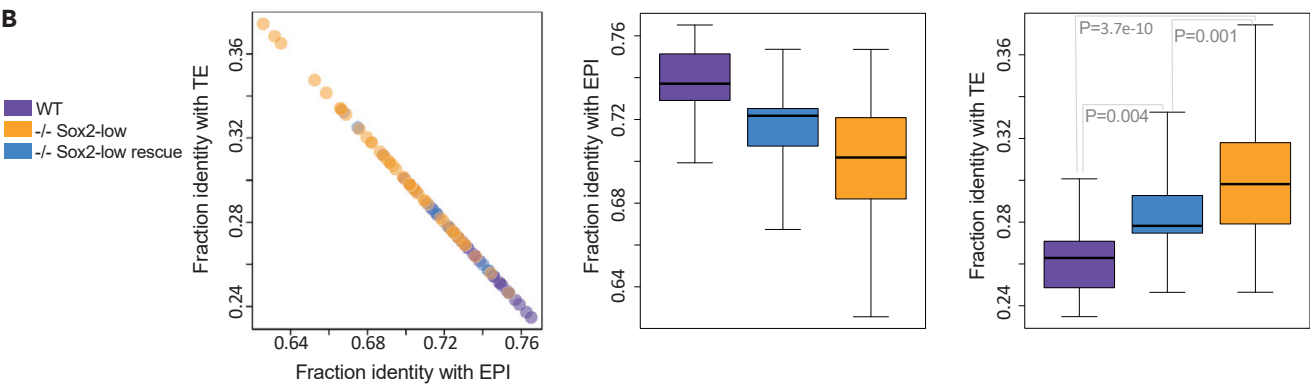

C

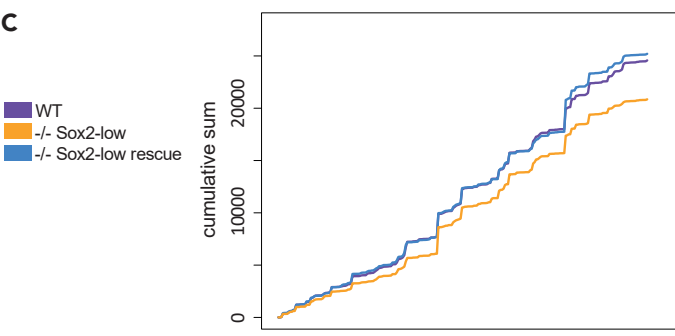

Genes upregulated in ICM

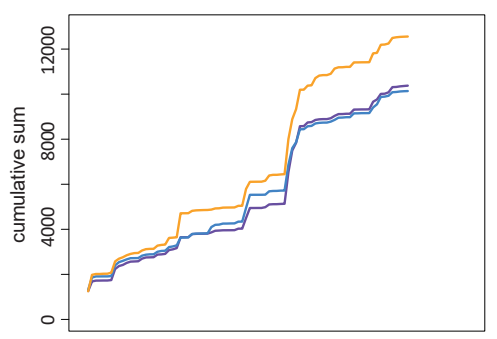

Genes upregulated in TE
D

Nanog proximal enhancer

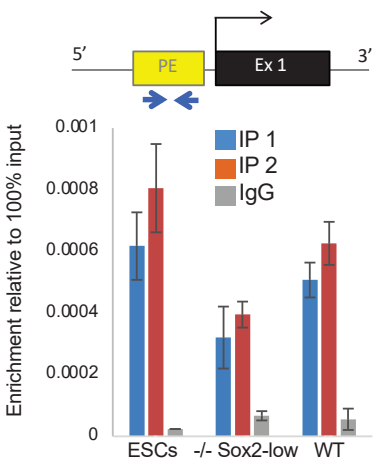

Oct4 distal enhancer

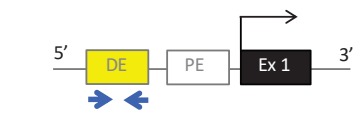

0.005

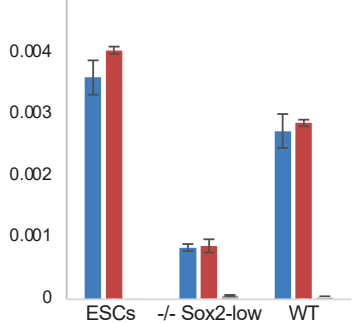

KIf2 proximal enhancer

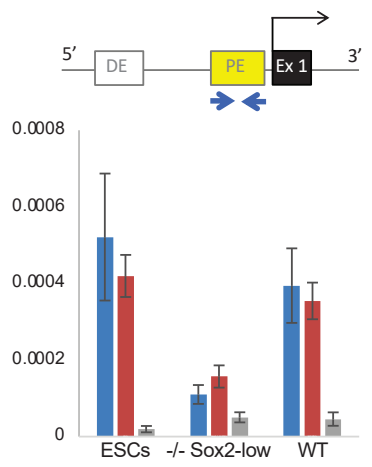

Figure 6. Sox2-low iPSCs have weaker epiblast identity

(A) Dendrogram computed with the top variable genes among the selected cell types (FPKM $>1, \log C V 2>0.5, n=1,446$ ) Trophectoderm/trophoblast (TE) E3.5 and E4.0 Single-cell RNA-sequencing (scRNA-seq) data is from (Deng et al., 2014), E4.5 Epiblast (Epi) scRNA-seq data is from (Mohammed et al., 2017), scRNA-seq ESC data is from (Sousa et al., 2018). (B) Scatterplot of fraction of identity between WT, -/- Sox2-low, -/- Sox2-low rescue iPSC line samples and embryo lineages (Epi and TE). Boxplot of the distribution of the fraction of identity between each population. Student's t-test was applied to calculate the significance of the differences in similarity to TE identity between -/-Sox2-low, WT and -l-Sox2-low rescue iPSCs.

(C) Cumulative sum for genes upregulated in TE or ICM (Blakeley et al., 2015), computed with gene expression value for WT, -I- Sox2-low, -/- Sox2-low rescue iPSC line samples. 
Figure 6. Continued

(D) Oct4 chromatin immunoprecipitation in Rex1-GFP ESCs and iPSCs in 2iLIF. IP = immunoprecipitation; IgG = negative control. Error bars represent the standard deviation of three technical qPCR replicates. IP1 and IP2 represent independent Oct4 immunoprecipitations. IgG represents negative control normal IgG immunoprecipitation.

from control lines (Figure S4A). Importantly, the rescue line clustered together with the parental line clearly indicating that the Sox2 expression level is the reason for grouping cells.

Gene ontology enrichment for genes differentially expressed between Sox2-low iPSCs and control iPSCs revealed only 325 differentially expressed genes between the different genotypes with no strongly enriched GO terms (Figures S4B and Data S1). In addition, no Sox family member gene was found upregulated in Sox2-low iPSCs, eliminating that way possible functional redundancy (Corsinotti et al., 2017). In agreement with Sox2-low iPSCs having a naive identity they clustered closely with control iPSCs, ESCs and E4.5 naive epiblast cells and separate from embryo trophoblast/trophectoderm (TE) cells (Figure 6A). However, when looking at the identity of single iPSCs on a continuum from an embryonic TE or naive epiblast perspective Sox2-low iPSCs were generally found in the fractions further away from the epiblast and nearer to the TE molecular identities relative to control iPSCs (Figure 6B). We also looked at accumulative gene expression for genes known to be associated with either the TE or with the ICM of embryos (Blakeley et al., 2015) (Figure 6C). Again, this revealed that Sox2-low iPSCs can be distinguished from WT and rescue lines as they display lower ICM and higher TE gene expression accumulation relative to control iPSCs.

ScRNA-seq analysis suggest that observed increased plasticity of Sox2-low cells is a functional property present at the single-cell level, as opposed to cell population heterogeneity.

\section{Reduced Sox2 expression impairs Oct4 DNA binding}

Sox2 is thought to be a DNA-binding partner to Oct4, and Oct4 inhibits extraembryonic differentiation (Niwa et al., 2000; Reményi et al., 2003). Therefore, we hypothesized that reduced Sox2 protein level may impact directly or indirectly on Oct4 DNA-binding ability in nPSC. Consistent with this notion, Oct4 chromatin immunoprecipitation showed reduced Oct4 genomic occupancy in Sox2-low iPSCs at key naive pluripotent regulatory sequences (Figure 6D).

These results suggest that reduced Oct4 occupancy at naive associated regulatory sequences combined with closer TE molecular identity relative to controls are likely underlying causes rendering Sox2-low iPSCs competent to also contribute to the trophoblast lineage.

\section{DISCUSSION}

Here we show that reduced levels of Sox2 are compatible with self-renewal of nPSCs in 2iL conditions, making these a good model to study the role of Sox 2 in early development.

These cells differentiate in SLIF demonstrating fragility in their pluripotent network. This fragility is likely due to the basal TE molecular identity and to the reduced Oct4 DNA-binding at naïve-associated regulatory sequences. Importantly, not only these cells can contribute toward the neural fate but also show increased cell plasticity, that is, they can differentiate into both embryonic and extraembryonic cell fates. This highlights a mechanism, modulation of Sox 2 expression to lower than $30 \%$ of WT levels, by which a nPSC is competent, upon differentiation signals, to contribute toward both embryonic and extraembryonic cell fates; previous studies only identified conditions that allowed cells to differentiate to one fate or the other (Masui et al., 2007; Nichols et al., 1998).

Sox2 and Oct4 have been hypothesized to bind together at Oct/Sox elements to drive the pluripotency network (Chew et al., 2005; Rodda et al., 2005). It has been shown that Sox2 binds to the DNA target sequence first and that it recruits Oct4 (Chen et al., 2014). However, Oct4 and Sox2 were also reported to operate in a largely independent manner and their impact on each others ability to bind to regulatory sequences may be indirect and regulated by chromatin accessibility (Friman et al., 2019). Independent of the Oct4-Sox2 relationship these studies are consistent with our findings that reduced Sox2 expression disrupts optimal Oct4 DNA-binding. 
We found that reduced or complete depletion of Sox2 is associated with differentiation into extraembryonic lineages, as well as the 3 germ layers. This indicates that Sox 2 plays a role in reducing the potency of nPSCs such that they cannot differentiate into extraembryonic lineages. The mechanism by which reduced Sox2 expression levels releases an inhibition on extraembryonic differentiation is likely linked to the impaired Oct4 genomic occupancy. Similar to Sox2 deletion, Oct4 deletion in ESCs causes differentiation into trophoblast-like cells (Niwa et al., 2000). However, unlike Sox2-low nPSCs, which can contribute to both embryonic and extraembryonic tissues, Oct4-low nPSCs lose the ability to differentiate into embryonic lineages (Radzisheuskaya et al., 2013). This suggests that in contrast to Oct4, Sox2 is not a key factor regulating differentiation toward embryonic lineages.

The importance of Sox 2 in inhibiting extraembryonic differentiation is counter to the evidence suggesting Sox 2 has a key role in extraembryonic development. Sox 2 is required to derive trophoblast stem cells and is expressed in extraembryonic tissues post-implantation (Avilion et al., 2003). However, this is likely to be an in vitro only requirement as Sox $2^{-1-}$ extraembryonic tissues are sufficient for development at least up to E12.5 (Avilion et al., 2003).

Morula aggregations using the Sox2-low iPSCs showed that only one out of 28 chimeric embryos exhibited trophectoderm contribution by the blastocyst stage. This is in stark contrast to E6.5 at which point $90 \%$ of all chimeric embryos display trophectoderm contribution. Interestingly, we observed cells within the epiblast upregulating the extraembryonic marker AP- $2 \gamma$ despite being also Oct4 positive. This is in agreement with Sox2-low iPSCs having initially a pluripotent identity, but upon differentiation showing additional competency to upregulate TE lineage markers and to contribute toward this lineage. Thus, their ability to undergo an extraembryonic lineage fate occurs after naive pluripotent cell identity exit and simultaneously with the embryonic lineage fate. The already reduced Oct4 genomic occupancy combined with the onset of the downregulation of naive factors upon initiation of cell differentiation may somehow create a window of opportunity for differentiating pluripotent cells to also acquire a TE fate.

Recently, expanded potential stem cells (EPSCs) that can contribute to both embryonic and extraembryonic lineages have been generated by two independent laboratories, by culturing ESCs in the presence of small molecules (Yang et al., 2017a, 2017b). The two laboratories used different chemical cocktails to generate the EPSCs but both resulted in cells with similar properties, suggesting a possible common mechanism. Among the added chemicals are minocycline hydrochloride (Yang et al., 2017b), a Parp1 inhibitor, and XAV939 (Yang et al., 2017a), a tankyrase inhibitor. The contributory mechanism of XAV939 to the extension of pluripotency is unclear, but it likely inhibits Parp family members TNKS1/2 and/or stabilizes AXIN (Yang et al., 2017a). Furthermore, Parp1 ${ }^{-1}$ ESCs have a propensity to differentiate into trophoblast (Hemberger et al., 2003). Parp1 is thought to aid Sox2 binding in ESCs (Liu and Kraus, 2017). Therefore, the previously published EPSC culture condition may reduce PARP activity and consequently reduce Sox2 DNA-binding, thus resulting in a similar phenotype to our Sox2-low nPSCs.

In conclusion, our study identifies an unexpected role of a naive pluripotency factor as a restrictor of developmental potency and provides a conciliatory mechanistic explanation for EPSCs, which also exhibit both embryonic and extraembryonic differentiation capacity. It will now be also interesting to investigate if a similar mechanism underlies recently described derivation of trophoblast cell derivatives directly from human nPSCs (Dong et al., 2020).

\section{Limitations of the study}

A caveat of our study concerns the lack of demonstration that generated trophoblast-like cells give rise to mature and functional trophoblast cell derivatives. We find the genotype of our cells not suitable to address this question as our cells express reduced levels of Sox2. This may cause multiple embryo phenotypes, as Sox2 is normally expressed in multiple tissues, including extraembryonic ones, which are likely to preclude long-term embryo survival. In addition, our work did not intend to create cells with extra potency but rather provide an explanation on how this may arise within a naive pluripotent stem identity.

\section{Resource availability}

\section{Lead contact}

Further information and requests should be directed to and will be fulfilled by the corresponding author, José C. R. Silva (jose_silva@grmh-gdl.cn). 
Materials availability

Please contact us if you would like to request any materials.

Data and code availability

The single-cell RNA-sequencing data generated during this study is available in the ArrayExpress repository under accession E-MTAB-9931.

\section{METHODS}

All methods can be found in the accompanying Transparent methods supplemental file.

\section{SUPPLEMENTAL INFORMATION}

Supplemental information can be found online at https://doi.org/10.1016/j.isci.2021.102153.

\section{ACKNOWLEDGMENTS}

We thank Yael Costa for critical reading of the manuscript. Peter Humphreys for assistance with imaging William Mansfield for blastocyst injections. This study was supported by a Wellcome Trust Fellowship (WT101861) to J.C.R.S. B.K.K. is supported by a European Research Council grant (639050). K.M. is a recipient of a Darwin Trust of Edinburgh Ph.D. studentship. K.T. is a recipient of an MRC Ph.D. studentship. H.T.S. and L.E.B. were supported by BBSRC and MRC research grants, BB/R018588/1 and MR/R017735/ 1 , respectively. G.G.S. is funded by BBSRC research grant RG53615.

\section{AUTHOR CONTRIBUTIONS}

J.C.R.S. conceived and supervised the study, designed experiments, wrote, and approved the manuscript. K.T. designed and performed experiments, analyzed the data and wrote the manuscript. A.R. initiated the study, designed experiments, and helped in the supervision. L.E.B. designed and performed the Oct4 ChIP experiment and other experiments. K.M. performed the neuroectoderm monolayer and other experiments. K.J. performed embryo dissections. A.A.R. generated Sox2 FLIP/FLIP ESCs overseen by B.K.K. H.T.S. designed and performed the single-cell sequencing experiment. G.G.S. and P.B. designed and performed bioinformatic analyses.

\section{DECLARATION OF INTERESTS}

The authors declare no competing interests.

Received: July 26, 2020

Revised: August 31, 2020

Accepted: February 2, 2021

Published: March 19, 2021

\section{REFERENCES}

Andersson-Rolf, A., Mustata, R.C., Merenda, A., Kim, J. Perera, S., Grego, T., Andrews, K. Tremble, K., Silva, J.C.R., Fink, J., et al. (2017) One-step generation of conditional and reversible gene knockouts. Nat. Methods 14 287-289.

Avilion, A.A., Nicolis, S.K., Pevny, L.H., Perez, L. Vivian, N., and Lovell-Badge, R. (2003). Multipotent cell lineages in early mouse development depend on SOX2 function. Genes Dev. 17, 126-140.

Blakeley, P., Fogarty, N.M.E., Del Valle, I., Wamaitha, S.E. Hu, T.X. Elder, K. Snell, P. Christie, L., Robson, P., and Niakan, K.K. (2015) Defining the three cell lineages of the human blastocyst by single-cell RNA-seq. Development 142, 3151-3165.
Chen, J., Zhang, Z., Li, L., Chen, B.C., Revyakin, A. Haji, B., Legant, W., Dahan, M., Lionnet, T., Betzig, E., et al. (2014). Single-molecule dynamics of enhanceosome assembly in embryonic stem cells. Cell 156, 1274-1285.

Chew, J.L., Loh, Y.H., Zhang, W., Chen, X., Tam, W.L., Yeap, L.S., Li, P., Ang, Y.S., Lim, B., Robson, $P$. et al. (2005). Reciprocal transcriptional regulation of Pou5f1 and Sox2 via the Oct4/Sox2 complex in embryonic stem cells. Mol. Cell Biol. $25,6031-6046$

Corsinotti, A., Wong, F.C.K., Tatar, T., Szczerbinska, I., Halbritter, F., Colby, D. Gogolok, S., Pantier, R., Liggat, K., Mirfazeli, E.S., et al. (2017). Distinct SoxB1 networks are required for naïve and primed pluripotency. Elife 6 e27746.
Deng, Q., Ramsköld, D., Reinius, B., and Sandberg, R. (2014). Single-cell RNA-seq reveals dynamic, random monoallelic gene expression in mammalian cells. Science 343, 193-196.

Dong, C., Beltcheva, M., Gontarz, P., Zhang, B., Popli, P., Fischer, L.A., Khan, S.A., Park, K.M. Yoon, E.J. Xing, X et al. (2020). Derivation of trophoblast stem cells from naïve human pluripotent stem cells. Elife 9, e52504.

Evans, M.J., and Kaufman, M.H. (1981). Establishment in culture of pluripotential cells from mouse embryos. Nature 292, 154-156.

Friman, E.T., Deluz, C., Meireles-Filho, A.C.A. Govindan, S., Gardeux, V., Deplancke, B., and Suter, D.M. (2019). Dynamic regulation of chromatin accessibility by pluripotency transcription factors across the cell cycle. Elife 8 e50087. 
Hemberger, M., Nozaki, T., Winterhager, E., Yamamoto, H., Nakagama, H., Kamada, N Suzuki, H., Ohta, T., Ohki, M., Masutani, M., et al. (2003). Parp1-deficiency induces differentiation of ES cells into trophoblast derivatives. Dev. Biol. $257,371-381$

Hotta, A., and Ellis, J. (2008). Retroviral vector silencing during iPS cell induction: an epigenetic beacon that signals distinct pluripotent states.

J. Cell Biochem. 105, 940-948.

Kopp, J.L., Ormsbee, B.D., Desler, M., and Rizzino, A. (2008). Small increases in the level of Sox2 trigger the differentiation of mouse embryonic stem cells. Stem Cells 26, 903-911.

Liu, Z., and Kraus, W.L. (2017). Catalyticindependent functions of PARP-1 determine Sox2 pioneer activity at intractable genomic loci. Mol. Cell 65, 589-603.e9.

Loh, K.M., and Lim, B. (2011). A precarious balance: pluripotency factors as lineage specifiers. Cell Stem Cell 8, 363-369.

Martin, G.R. (1981). Isolation of a pluripotent cell line from early mouse embryos cultured in medium conditioned by teratocarcinoma stem cells. Proc. Natl. Acad. Sci. U S A 78, 7634-7638.

Masui, S., Nakatake, Y., Toyooka, Y., Shimosato, D., Yagi, R., Takahashi, K., Okochi, H., Okuda, A., Matoba, R. Sharov, A.A., et al. (2007)

Pluripotency governed by Sox2 via regulation of Oct3/4 expression in mouse embryonic stem cells. Nat. Cell Biol. 9, 625-635.

Mohammed, H., Hernando-Herraez, I., Savino, A. Scialdone, A., Macaulay, I., Mulas, C., Chandra

T. Voet, T. Dean, W., Nichols, J., et al. (2017). Single-cell landscape of transcriptional heterogeneity and cell fate decisions during mouse early gastrulation. Cell Rep. 20,12151228 .
Nichols, J., Zevnik, B., Anastassiadis, K., Niwa, H., Klewe-Nebenius, D. Chambers, I. Schöler, $H_{\text {. }}$ and Smith, A. (1998). Formation of pluripotent stem cells in the mammalian embryo depends on the POU transcription factor Oct4. Cell 95, 379-391.

Niwa, H., Miyazaki, J., and Smith, A.G. (2000). Quantitative expression of Oct-3/4 defines differentiation, dedifferentiation or self-renewal of ES cells. Nat. Genet. 24, 372-376.

Radzisheuskaya, A., Chia Gle, B., dos Santos, R.L. Theunissen, T.W., Castro, L.F.C., Nichols, J., Silva J.C.R. Bin Chia, G.L., dos Santos, R.L.,

Theunissen, T.W., et al. (2013). A defined Oct4 level governs cell state transitions of pluripotency entry and differentiation into all embryonic lineages. Nat. Cell Biol. 15, 579-590.

Reményi, A., Lins, K., Nissen, L.J., Reinbold, R. Schöler, H.R., Wilmanns, M. Remenyi, A. Lins, K. Nissen, L.J., Reinbold, R., et al. (2003). Crystal structure of a POU/HMG/DNA ternary complex suggests differential assembly of Oct4 and Sox2 on two enhancers. Genes Dev. 17, 2048-2059.

Rodda, D.J., Chew, J.L., Lim, L.H., Loh, Y.H., Wang, B., Ng, H.H., and Robson, P. (2005). Transcriptional regulation of Nanog by OCT4 and SOX2. J. Biol. Chem. 280, 24731-24737.

Silva, J., Barrandon, O., Nichols, J., Kawaguchi, J. Theunissen, T.W., and Smith, A. (2008)

Promotion of reprogramming to ground state pluripotency by signal inhibition. PLoS Biol. 6, e253.

Sousa, E.J., Stuart, H.T., Bates, L.E., Ghorbani, M. Nichols, J., Dietmann, S., and Silva, J.C.R. (2018) Exit from naive pluripotency induces a transient $X$ chromosome inactivation-like state in males. Cel Stem Cell 22, 919-928.e6.

Takahashi, K., and Yamanaka, S. (2006). Induction of pluripotent stem cells from mouse embryonic and adult fibroblast cultures by defined factors. Cell 126, 663-676.

Thomson, M., Liu, S.J., Zou, L.N., Smith, Z., Meissner, A., and Ramanathan, S. (2011).

Pluripotency factors in embryonic stem cells regulate differentiation into germ layers. Cell 145, 875-889.

Wray, J., Kalkan, T., Gomez-Lopez, S., Eckardt, D. Cook, A., Kemler, R., and Smith, A. (2011). Inhibition of glycogen synthase kinase-3 alleviates $\mathrm{Tcf} 3$ repression of the pluripotency network and increases embryonic stem cell resistance to differentiation. Nat. Cell Biol. 13, 838-845.

Wright, E.M., Snopek, B., and Koopman, P. (1993). Seven new members of the Sox gene during mouse development family expressed during mouse development. Nucl. Acids Res. 21, 744.

Yang, J., Ryan, D.J., Wang, W., Tsang, J.C.-H. Lan, G., Masaki, H., Gao, X., Antunes, L., Yu, Y. Zhu, Z., et al. (2017a). Establishment of mouse expanded potential stem cells. Nature 550, 393-397.

Yang, Y., Liu, B., Xu, J., Wang, J., Wu, J., Shi, C. Xu, Y., Dong, J., Wang, C., Lai, W., et al. (2017b). Derivation of pluripotent stem cells with in vivo embryonic and extraembryonic potency. Cell 169, 243-257.e5.

Ying, Q.-L., Stavridis, M., Griffiths, D., Li, M., and Smith, A. (2003). Conversion of embryonic stem cells into neuroectodermal precursors in adherent monoculture. Nat. Biotechnol. 21, 183-186.

Yuan, H., Corbi, N., Basilico, C., and Dailey, L. (1995). Developmental-specific activity of the FGF-4 enhancer requires the synergistic action of Sox2 and Oct-3. Genes Dev. 9, 2635-2645. 
iScience, Volume 24

\section{Supplemental information}

Sox2 modulation increases

naïve pluripotency plasticity

Kathryn C. Tremble, Giuliano G. Stirparo, Lawrence E. Bates, Katsiaryna Maskalenka, Hannah T. Stuart, Kenneth Jones, Amanda Andersson-Rolf, Aliaksandra Radzisheuskaya, Bon-Kyoung Koo, Paul Bertone, and José C.R. Silva 


\section{Transparent methods}

\section{Plasmids}

pMXs c-Myc, pMXs Oct4, pMXs Sox2, pMXs Klf4 and pSpCas9(BB)-2A-GFP (Addgene). pPyCAGMST-IRES-Puro, pPyCAG-eGFP-IRES-Zeo (Austin Smith). pPB-CAG-DEST-hygro, pCAGCreERT2NLS-IRES-bsd (Joerg Betschinger). PB-CAG-Sox2-hygro was used for reprogramming and for the rescue of Sox2-low iPSCs.

\section{Cell culture}

PLAT-E cells and (where stated) iPSCs were cultured in GMEM basal medium (GMEM (Sigma-Aldrich), 1xNEAA (Gibco), 1xpenicillin-streptomycin (Sigma-Aldrich), 1mM sodium pyruvate (Sigma-Aldrich), $0.1 \mathrm{mM}$ 2-mercaptoethanol (ThermoFisher Scientific) and $2 \mathrm{mM}$ L-glutamine (ThermoFisher Scientific)) with 10\% FCS (Labtech) and 20ng/ml mouse LIF (University of Cambridge). NSCs were cultured in DMEM/F12 (ThermoFisher Scientific), 1xNEAA, 0.1mM 2-mercaptoethanol, 1xpenicillin-streptomycin, 1:100 v/v B27 supplement (ThermoFisher Scientific), 1:200 v/v N2 supplement (University of Cambridge), $4.5 \mu \mathrm{M}$ HEPES (ThermoFisher Scientific), $0.03 \mathrm{M}$ glucose and $120 \mu \mathrm{g} / \mathrm{ml}$ BSA (ThermoFisher Scientific) supplemented with 10ng/ml EGF (Peprotech) and 20ng/ml FGF2 (University of Cambridge). iPSCs were cultured in KSR basal medium (GMEM basal medium with $10 \% \mathrm{KSR}, 1 \%$ FCS) or N2B27 basal medium (DMEM/F12 and Neurobasal (ThermoFisher Scientific) in a 1:1 ratio, 1xpenicillin-streptomycin, 0.1mM 2-mercaptoethanol, 2mM L-glutamine, 1:200 v/v N2 and 1:100 v/v

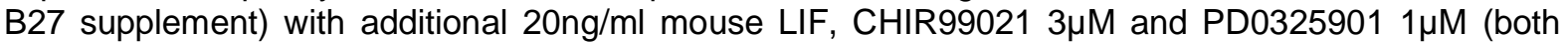
Biotechnology Center TU Dresden, Stewart lab). Additional chemicals used: hygromycin B $200 \mu \mathrm{g} / \mathrm{ml}$ (ThermoFisher Scientific), blasticidin $40 \mu \mathrm{g} / \mathrm{ml}$ (ThermoFisher Scientific), puromycin $1 \mu \mathrm{g} / \mathrm{ml}$ (ThermoFisher Scientific), zeocin $100 \mu \mathrm{g} / \mathrm{ml}$ (ThermoFisher Scientific), 4-hydroxytamoxifen $500 \mathrm{nM}$.

\section{Reprogramming}

Separate PLAT-E cultures were transfected with $9 \mu \mathrm{g}$ of each pMX plasmid using FuGENE 6 (Roche) for retroviral production. 48 hours later the supernatants were collected, combined, and mixed with $4 \mu \mathrm{g} / \mathrm{ml}$ Polybrene (Sigma-Aldrich) and filtered through a $0.45 \mu \mathrm{m}$ cellulose acetate filter. This was applied to NS cells for 24 hours, after which they were returned to NS medium for 2 days, before being placed in serum/LIF to form reprogramming intermediates (preiPSCs). These were then placed in KSR2iLIF to form iPSCs.

\section{Cell differentiation}

For embryoid body assays, $1.5 \times 10^{6}$ cells were plated in suspension into serum basal media without LIF in $90 \mathrm{~mm}$ low-attachment dishes for 7 days with media changed every other day.

\section{Neural differentiation}

$1 \times 10^{5}$ cells were plated directly into 6 -well plates, previously coated with laminin for 2 hrs at 37 degrees, containing N2B27 plus $1 \mu \mathrm{M}$ Alk inhibitor (A83-01) differentiation medium and then cultured in a low oxygen incubator. Medium was changed every day.

\section{Western blotting}

The primary antibodies used were: rat monoclonal against Sox2 (1:2000, eBioscience, 149811); mouse monoclonal against $\alpha$-tubulin (1:10000, Abcam, ab7291). The secondary antibodies were HRP-linked antibodies against rat or mouse IgG (GE Healthcare).

\section{Embryo staining}

Embryos were fixed with 4\% paraformaldehyde and mounted in vectashield antifade mounting medium (Vector Laboratories). Blastocysts were permeabilised using 100\% methanol and blocked using $2 \%$ donkey serum, $0.1 \%$ BSA and $0.2 \%$ Triton $\mathrm{X}-100$. Post-implantation embryos were permeabilised in $0.25 \%$ Triton X-100/PBS and blocked using $0.01 \%$ Triton X-100/PBS and $3 \%$ donkey serum. Primary antibodies used were goat polyclonal against Sox17 (1:500, R and D systems, AF1924), mouse monoclonal against Cdx2 (1:500, Biogenex, MU392A-UC), AP-2y (1:100, Cell Signalling Technology, 2320), rat monoclonal against GFP (1:200, Nacalai, 04404-26), mouse monoclonal against Oct4 (1:100, Santa Cruz, sc-5279). Secondary antibodies were Alexa Fluor antibodies (ThermoFisher Scientific) and were used at a concentration of 1:500.

\section{Morula aggregation}


E2.5 CD1 embryos were combined with iPSCs and transferred to recipient mice to assess the contribution in post-implantation development or were cultured in vitro for 2 days to assess blastocyst contribution.

\section{Cell transfection}

Nucleofection (NSCs) was performed using the AMAXA Nucleofection Technology (Lonza, VCA-1003). Lipofection (ESCs, iPSCs) was performed using Lipofectamine 2000 Transfection Reagent (ThermoFisher Scientific).

\section{Western Blot Quantification}

Quantification was performed using the 'Gels' tool in ImageJ Fiji. Background-subtracted relative intensity (BSRI) was calculated for each band of interest. Protein of interest (Sox2) BSRI values were then normalized to the corresponding loading control (tubulin) BSRI values and ESCs.

\section{Derivation of Rex1-GFP Neural Stem cells}

Rex1dGFP.IRES.bsd/dGFP.IRES.bsd homozygous 129 studs (Kalkan et al., 2017) were crossed with wild-type 129 females and heterozygous Rex $1^{+/ d G F P . I R E S . b s d}$ Neural Stem cells (referred to as Rex1-GFP reporter) were derived from resultant E13.5 embryos as previously described (Pollard et al., 2006). Rex1-GFP Neural Stem cells have a 129 strain genetic background.

\section{Generation of CRISPR/Cas9 cell lines}

Sox2 $2^{-1 S C s}$ were generated by nucleofecting NSCs with $4 \mu \mathrm{g}$ CRISPR/gRNA plasmid and single-cell sorting 48 hours later to generate clonal cell lines. The generation of Sox2 FLIP/FLIP ESCs has been previously described (Andersson-Rolf et al., 2017). Sox2 FLIP/FLIP ESCs have a 129 strain genetic background.

\section{Chromatin Immunoprecipitation (ChIP)}

ChIP was performed as previously described (Radzisheuskaya et al., 2013) with minor changes. Briefly, $1 \times 10^{7}$ cells were fixed for $10 \mathrm{~min}$ in $0.4 \%$ formaldehyde then washed with ice-cold PBS. Cells were incubated in lysis buffer 1 (50 mM HEPES pH 7.5, $140 \mathrm{mM} \mathrm{NaCl}, 1 \mathrm{mM}$ EDTA, 10\% Glycerol, 0.5\% NP40, 0.25\% Tx100) then in lysis buffer 2 (10 mM Tris pH 8.0, $200 \mathrm{mM} \mathrm{NaCl}, 1 \mathrm{mM}$ EDTA, $0.5 \mathrm{mM}$ EGTA) for 10 min each. Nuclei were resuspended in shearing buffer (1\% SDS, $10 \mathrm{mM}$ EDTA, $50 \mathrm{mM}$ Tris $\mathrm{pH} 8.0$ ) and sonicated to an average fragment size of 300-500 bp. Chromatin was diluted 1:10 in dilution buffer (50 mM Tris pH 8.0, $167 \mathrm{mM} \mathrm{NaCl}, 1.1 \%$ Tx100, 0.11\% Na-deoxycholate) and cleared with isotype IgG coated protein G Dynabeads (ThermoFisher Scientific). A portion of chromatin was taken as input control. Chromatin was then incubated overnight at $4^{\circ} \mathrm{C}$ with $1.5 \mu \mathrm{g}$ Rabbit anti-Oct4 antibody (Abcam; ab19857) antibody or $2 \mu \mathrm{g}$ Rabbit normal IgG (Santa Cruz Biotechnology; sc-2027). Chromatin-antibody mix was incubated with pre-blocked protein $\mathrm{G}$ dynabeads for 1 hour at $4^{\circ} \mathrm{C}$. These were then washed twice in low salt wash buffer (50 mM Tris pH 8.0, 0.1\% SDS, $0.1 \%$ Na-deoxycholate, $1 \%$ Tx100, $150 \mathrm{mM} \mathrm{NaCl}, 1 \mathrm{mM}$ EDTA, $0.5 \mathrm{mM}$ EGTA), once in high salt wash buffer (50 mM Tris pH 8.0, $0.1 \%$ SDS, $0.1 \%$ Na-deoxycholate, $1 \%$ Tx100, $500 \mathrm{mM} \mathrm{NaCl}, 1 \mathrm{mM}$ EDTA, $0.5 \mathrm{mM}$ EGTA), once in $\mathrm{LiCl}$ wash buffer (50 mM Tris pH 8.0, $250 \mathrm{mM} \mathrm{LiCl}, 0.5 \%$ Na-deoxycholate, 0.5\% NP40, $1 \mathrm{mM}$ EDTA, $0.5 \mathrm{mM}$ EGTA) and twice in TE wash buffer ( $50 \mathrm{mM}$ Tris pH 8.0, $10 \mathrm{mM}$ EDTA, $5 \mathrm{mM}$ EGTA). Bound chromatin was then eluted at $65^{\circ} \mathrm{C}$ in elution buffer (1\% SDS, $0.1 \mathrm{M} \mathrm{NaHCO}_{3}$ ). Crosslinking was reversed through overnight incubation at $65^{\circ} \mathrm{C}$ for samples and inputs. DNA was purified using the QIAquick PCR Purification Kit (Qiagen) according to the manufacturer's protocol. DNA was quantitated by SYBR Green (ThermoFisher Scientific) qPCR, with a standard curve to ensure linear amplification. Immunoprecipitation efficiency was calculated relative to input $=1$.

\section{Primers for ChIP}

\begin{tabular}{|l|l|}
\hline Primer & Sequence \\
\hline Nanog proximal enhancer forward & GCAGCCGTGGTTAAAAGATG \\
\hline Nanog proximal enhancer reverse & GAAGCTGTAAGGTGACCCAGA \\
\hline Oct4 distal enhancer forward & GCATAACAAAGGTGCATGATAGCT \\
\hline Oct4 distal enhancer reverse & AAATAAAGGCAGCGACTTGGAA \\
\hline Klf2 proximal enhancer forward & CTGCACAAAGGGCTTAGAGG \\
\hline Klf2 proximal enhancer reverse & CCTCATTTGCACCACACCTA \\
\hline
\end{tabular}

RNA extraction, cDNA synthesis and qPCR 
Total RNA was isolated using the RNeasy Mini kit (QIAGEN). 1 $\mu \mathrm{g}$ RNA was reverse-transcribed using SuperScript III First-Strand Synthesis SuperMix for qRT-PCR (ThermoFisher Scientific). The resultant cDNA was analysed by quantitative PCR using TaqMan Fast Universal PCR Master Mix (ThermoFisher Scientific) with TaqMan Gene Expression Assays and/or KiCqStart assays, or with Fast SYBR Green Master Mix (ThermoFisher Scientific) using primers. qRT-PCR experiments were performed in triplicate on a StepOnePlus Real-Time PCR System (Applied Biosystems). Delta Ct values were normalised to GAPDH and raised to the power of -2 . Standard deviations refer to technical replicates.

\begin{tabular}{|c|c|}
\hline \multicolumn{2}{|c|}{ Primers used with SYBR Green } \\
\hline GAPDH FP & CCCACTAACATCAAATGGGG \\
\hline GAPDH RP & CCTTCCACAATGCCAAAGTT \\
\hline Olig2 FP & CTGCTGGCGCGAAACTACAT \\
\hline Olig2 RP & CGCTCACCAGTCGCTTCAT \\
\hline BLBP FP & AGACCCGAGTTCCTCCAGTT \\
\hline BLBP RP & ATCACCACTTTGCCACCTTC \\
\hline FoxA1 FP & ATGAGAGCAACGACTGGAACA \\
\hline FoxA1 RP & TCATGGAGTTCATAGAGCCCA \\
\hline Pl-1 FP & ATTTTGACTACCCTGCTTGGTCT \\
\hline Pl-1 RP & TCTACATAACTGAGGAGGGGAAAG \\
\hline Hand1 FP & CCCCTCTTCCGTCCTCTTAC \\
\hline Hand1 RP & CTGCGAGTGGTCACACTGAT \\
\hline Eomes FP & CCTGGTGGTGTTTTGTTGTG \\
\hline Eomes RP & TTTAATAGCACCGGGCACTC \\
\hline \multicolumn{2}{|c|}{ ThermoFisher custom Taqman probes } \\
\hline Endogenous Oct4 FP & TTCCACCAGGCCCCC \\
\hline Endogenous Oct4 RP & GGTGAGAAGGCGAAGTCTGAAG \\
\hline Endogenous Oct4 probe & FAM-CCCACCTTCCCCATGGCT-MGB \\
\hline Retroviral Sox2 FP & TGGTACGGGAAATCACAAGTTTGTA \\
\hline Retroviral Sox2 RP & GCCCGGCGGCTTCA \\
\hline Retroviral Sox2 probe & FAM-CTCCGTCTCCATCATGTTAT-MGB \\
\hline Retroviral cMyc FP & TGGTACGGGAAATCACAAGTTTGTA \\
\hline Retroviral cMyc RP & GGTCATAGTTCCTGTTGGTGAAGTT \\
\hline Retroviral cMyc probe & FAM-CCCTTCACCATGCCCC-MGB \\
\hline Retroviral Klf4 FP & TGGTACGGGAAATCACAAGTTTGTA \\
\hline Retroviral Klf4 RP & GAGCAGAGCGTCGCTGA \\
\hline Retroviral Klf4 probe & FAM-CCCCTTCACCATGGCTG-MGB \\
\hline Retroviral Oct4 FP & TGGTACGGGAAATCACAAGTTTGTA \\
\hline Retroviral Oct4 RP & GGTGAGAAGGCGAAGTCTGAAG \\
\hline Retroviral Oct4 probe & FAM-CACCTTCCCCATGGCTG-MGB \\
\hline \multicolumn{2}{|c|}{ Sigma-Aldrich KiCqStart Probes } \\
\hline Krt7 & MMUS_NM_033073_1 \\
\hline \multicolumn{2}{|c|}{ ThermoFisher Taqman Probes } \\
\hline Total Oct4 & $\mathrm{Mm00658129 \_ gH}$ \\
\hline GAPDH & 4352339E \\
\hline Nanog & Mm02384862_g1 \\
\hline Esrrb & Mm00442411_m1 \\
\hline FGF5 & Mm00438918_m1 \\
\hline T Brachyury & Mm01318252_m1 \\
\hline Gata4 & $\mathrm{Mm00484689 \_ m1}$ \\
\hline KIf2 & Mm01244979_g1 \\
\hline Sox1 & Mm00486299_s1 \\
\hline
\end{tabular}




\begin{tabular}{|l|l|}
\hline Ascl1 & Mm03058063_m1 \\
\hline Pax6 & Mm00443081_m1 \\
\hline Zeb2 & Mm00497193_m1 \\
\hline Elf5 & Mm00468732_m1 \\
\hline
\end{tabular}

\section{ScRNA-seq library preparation}

Single cells were index-sorted individually by FACS (BD Influx 5) into wells of a 96-well PCR plate containing lysis buffer. ScRNA-seq was performed as previously described (Nestorowa et al., 2016; Picelli et al., 2014; Wilson et al., 2015). The Illumina Nextera XT DNA kit was used to prepare libraries. Pooled libraries were sequenced on the Illumina HiSeq 4000 (single-end 125bp reads).

\section{RNA-seq data analysis}

Sequencing reads were aligned to mouse genome reference $\mathrm{GRCm} 38 / \mathrm{mm} 10$ with STAR (Dobin et al., 2013) using the two-pass method for novel splice detection (Engström et al., 2013). GENCODE M12 mouse gene annotation from Ensembl release 87 (Yates et al., 2016) was used for read alignment and splice junction donor/acceptor overlap settings were tailored to the read length of each dataset. Alignments to gene loci were quantified with HTSeq-count (Anders et al., 2015) based on annotation from Ensembl release 87. Quality control was performed according to (Stirparo et al., 2018). Briefly, sequencing libraries with fewer than 500,000 mapped reads were excluded from subsequent analyses. Read distribution bias across gene bodies was computed as the ratio between the total reads spanning the 50th to the 100th percentile of gene length, and those between the first and 49th. Samples with ratio $>2$ were not considered further. Stage-specific outliers were screened by principal component analysis.

\section{Published scRNA-seq datasets}

Sequencing data corresponding to single-cell mouse embryo profiling studies SRP110669 (Mohammed et al., 2017) (E3.5, E4.5), SRP020490 (Deng et al., 2014) (trophectoderm cells) and E-MTAB-7901 (Stuart et al., 2019) (ESCs) were obtained from the European Nucleotide Archive (Toribio et al., 2017) and from ArrayExpress repository and processed as above.

\section{Transcriptome analysis}

Principal component and cluster analyses were performed based on log2 FPKM values and were computed with FactoMineR (Lê et al., 2008) in addition to custom scripts. Default parameters were used unless otherwise indicated. For global analyses, genes that registered zero counts in all singlecell samples were omitted. Euclidean distance and complete linkage were used for cluster analyses unless otherwise indicated. Differential expression analysis was performed with scda (Kharchenko et al., 2014), that fits individual error models for assessment of differential expression between groups of cells. DAVID Bioinformatics Resources 6.7 (Huang et al., 2009) was used for computing the enriched biological processes, using as input list the modulated genes (with padj value $<0.05$ ) between mutant cells and wt/rescue cells. Genes exhibiting the greatest expression variability (and thus contributing substantial discriminatory power) were identified by fitting a non-linear regression curve between average log2 FPKM and the square of coefficient of variation. Indicated specific thresholds were applied along the $x$-axis (average log2 FPKM) and $y$-axis (CV2) to identify the most variable genes. Cumulative sum was computed by performing the sequential sums of log2 expression values for genes expressed in TE or ICM stage.

\section{Data and Code Availability}

The single-cell RNA sequencing data generated during this study is available in the ArrayExpress repository under accession E-MTAB-9931 


\section{Supplemental References}

Anders, S., Pyl, P.T., and Huber, W. (2015). HTSeq-A Python framework to work with high-throughput sequencing data. Bioinformatics 31, 166-169.

Andersson-Rolf, A., Mustata, R.C., Merenda, A., Kim, J., Perera, S., Grego, T., Andrews, K., Tremble, K., Silva, J.C.R., Fink, J., et al. (2017). One-step generation of conditional and reversible gene knockouts. Nat Methods.

Deng, Q., Ramsköld, D., Reinius, B., and Sandberg, R. (2014). Single-cell RNA-seq reveals dynamic, random monoallelic gene expression in mammalian cells. Science (80- ) 343, 193-196.

Dobin, A., Davis, C.A., Schlesinger, F., Drenkow, J., Zaleski, C., Jha, S., Batut, P., Chaisson, M., and Gingeras, T.R. (2013). STAR: Ultrafast universal RNA-seq aligner. Bioinformatics.

Engström, P.G., Steijger, T., Sipos, B., Grant, G.R., Kahles, A., Rätsch, G., Goldman, N., Hubbard, T.J., Harrow, J., Guigó, R., et al. (2013). Systematic evaluation of spliced alignment programs for RNA-seq data The RGASP Consortium Europe PMC Funders Group. Nat Methods.

Huang, D.W., Sherman, B.T., and Lempicki, R.A. (2009). Systematic and integrative analysis of large gene lists using DAVID bioinformatics resources. Nat Protoc.

Kalkan, T., Olova, N., Roode, M., Mulas, C., Lee, H.J., Nett, I., Marks, H., Walker, R., Stunnenberg, H.G., Lilley, K.S., et al. (2017). Tracking the embryonic stem cell transition from ground state pluripotency. Development 144, 1221-1234.

Kharchenko, P. V., Silberstein, L., and Scadden, D.T. (2014). Bayesian approach to single-cell differential expression analysis. Nat Methods 11, 740-742.

Lê, S., Josse, J., and Husson, F. (2008). FactoMineR: An R Package for Multivariate Analysis. J Stat Softw 25, 253-258.

Mohammed, H., Hernando-Herraez, I., Savino, A., Scialdone, A., Macaulay, I., Mulas, C., Chandra, T., Voet, T., Dean, W., Nichols, J., et al. (2017). Single-Cell Landscape of Transcriptional

Heterogeneity and Cell Fate Decisions during Mouse Early Gastrulation. Cell Rep 20, 1215-1228.

Nestorowa, S., Hamey, F.K., Pijuan Sala, B., Diamanti, E., Shepherd, M., Laurenti, E., Wilson, N.K., Kent, D.G., and Göttgens, B. (2016). A single cell resolution map of mouse haematopoietic stem and progenitor cell differentiation. Blood blood-2016-05-716480.

Picelli, S., Faridani, O.R., Björklund, Å.K., Winberg, G., Sagasser, S., and Sandberg, R. (2014). Fulllength RNA-seq from single cells using Smart-seq2. Nat Protoc 9, 171-181.

Pollard, S.M., Conti, L., Sun, Y., Goffredo, D., and Smith, A. (2006). Adherent neural stem (NS) cells from fetal and adult forebrain. Cereb Cortex 16.

Radzisheuskaya, A., Chia Gle, B., dos Santos, R.L., Theunissen, T.W., Castro, L.F.C., Nichols, J., Silva, J.C.R., Chia, G.L. Bin, dos Santos, R.L., Theunissen, T.W., et al. (2013). A defined Oct4 level governs cell state transitions of pluripotency entry and differentiation into all embryonic lineages. Nat Cell Biol 15, 579-590.

Stirparo, G.G., Boroviak, T., Guo, G., Nichols, J., Smith, A., and Bertone, P. (2018). Integrated analysis of single-cell embryo data yields a unified transcriptome signature for the human preimplantation epiblast. Dev.

Stuart, H.T., Stirparo, G.G., Lohoff, T., Bates, L.E., Kinoshita, M., Lim, C.Y., Sousa, E.J., Maskalenka, K., Radzisheuskaya, A., Malcolm, A.A., et al. (2019). Distinct Molecular Trajectories Converge to Induce Naive Pluripotency. Cell Stem Cell 25, 388-406.e8.

Wilson, N.K., Kent, D.G., Buettner, F., Shehata, M., Macaulay, I.C., Calero-Nieto, F.J., Sánchez Castillo, M., Oedekoven, C.A., Diamanti, E., Schulte, R., et al. (2015). Combined Single-Cell Functional and Gene Expression Analysis Resolves Heterogeneity within Stem Cell Populations. Cell Stem Cell 16, 712-724.

Yates, A., Akanni, W., Amode, M.R., Barrell, D., Billis, K., Carvalho-Silva, D., Cummins, C., Clapham, P., Fitzgerald, S., Gil, L., et al. (2016). Ensembl 2016. Nucleic Acids Res 44, D710-D716. 
A Sox2 DNA sequencing WT

Sox2 Predicted Protein Sequence

WT MYNMMETELKPPGPQQASGGGGGGGNATAAATGGNQKNSPDRVKRPM..........

SOX2-/- Cl 3 MYNMMETELKPPARSKLRGAAAEEATPRRRRPAATRRTARTASRGP*

B

Sox2 gRNA CRISPR Clones

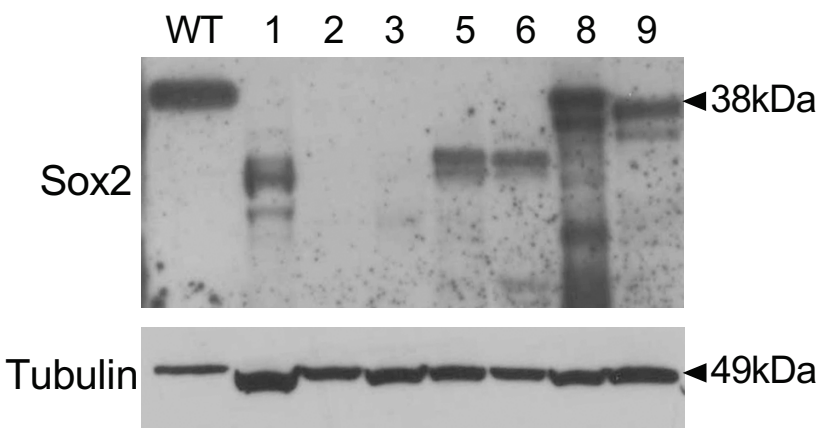

C

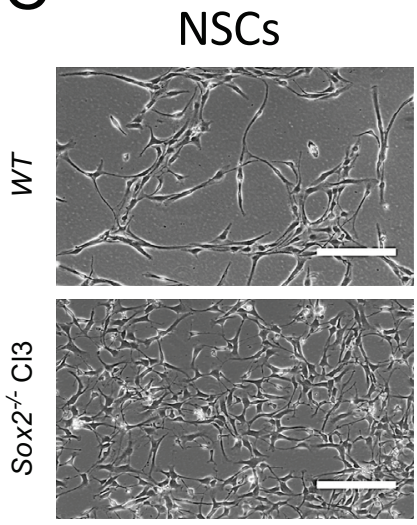

$\mathrm{D}$

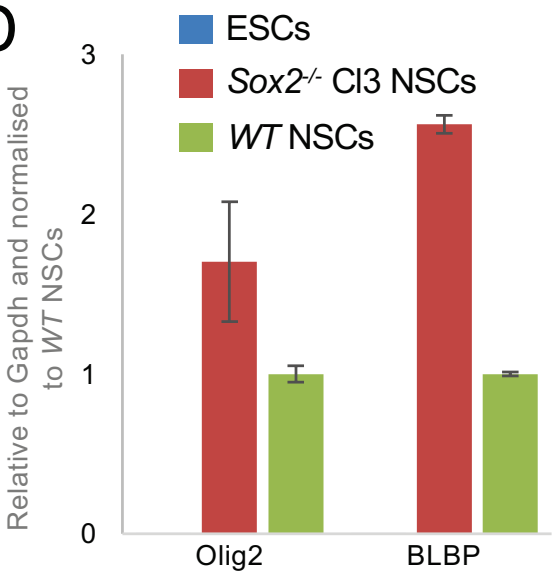

Figure S1- Generation of Sox2-/ NSCs. Related to Figure 1.

A) Sequence of start of coding region of Sox2 amplified by genomic PCR for Sox2-- neural stem cell (NSC) clone 3 with Cas9 targeting gRNA binding site underlined aligned with the WT Sox2 coding region to show the mutation (deletion of 1 guanine). The predicted protein sequence from the Sox2- NSC clone 3 is shown aligned with WT sequence, with the sequence diversion highlighted. ${ }^{*}=$ stop codon.

B) Western blot for Sox2 $(\approx 40 \mathrm{kDa})$ and Tubulin $(\approx 50 \mathrm{kDa})$ protein expression in WT and clonal NSC lines after transfection with Sox2 gRNA/Cas9.

C) Phase images of WT and Sox2- clone (Cl) 3 NSCs.

D) qRT-PCR analysis of neural markers (Olig2 and BLBP) in WT and Sox2- Cl3 NSCs and ESCs. Error bars indicate standard deviation of replicate qPCR reactions $(n=3)$.

Scale bars $=200 \mu \mathrm{m}$. 


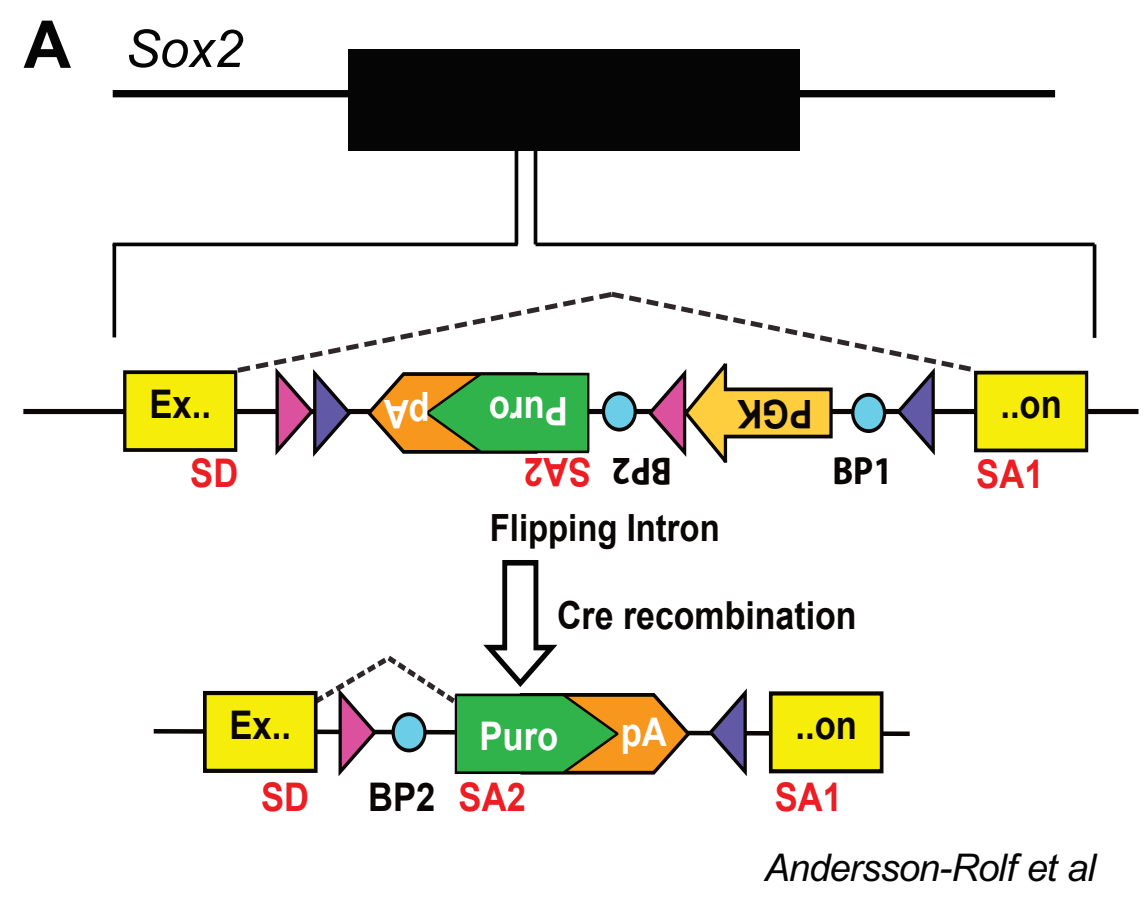

B
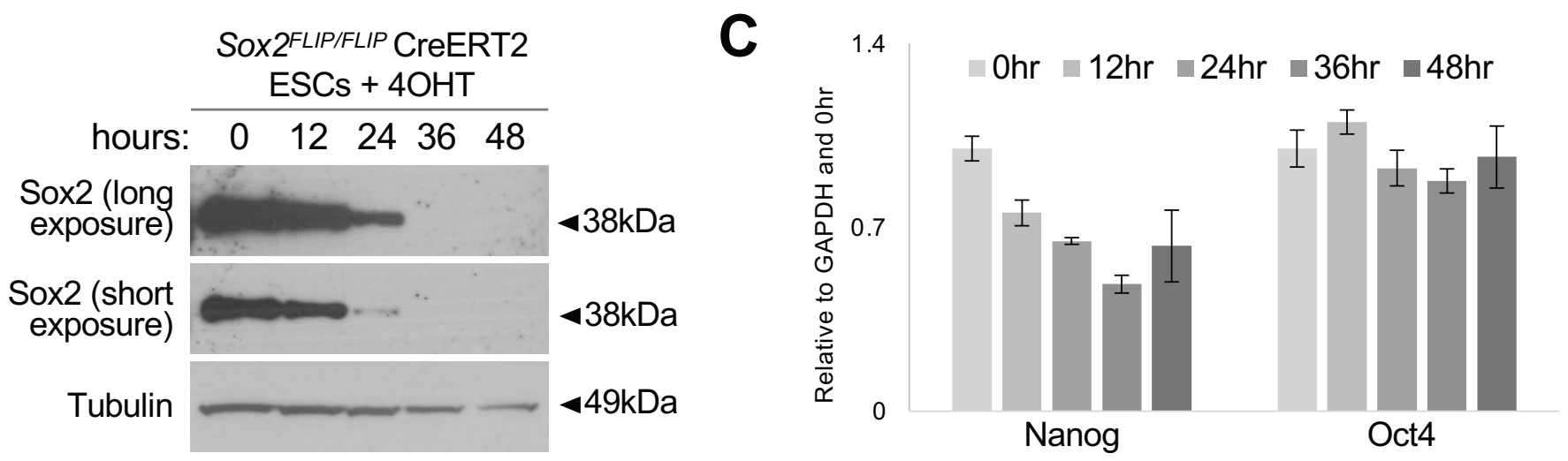

Figure S2- Sox2FLIPIFLIP ESCs experimental optimisation. Related to Figure 3.

A) Schematic of Sox2FLIP/FLIP ESCs. Please note that after Cre mediated recombination Sox2 protein becomes truncated. SD indicates splice donor site. BP1 and SA1, and BP2 and SA2 indicate pairs of branch points and splice acceptor sites that are used before and after Cre recombination respectively. Pink and purple triangles indicate LoxP variants (Lox5171 and LoxP1 respectively). Dashed line indicates splice site usage.

B) Western blot of 48 hour timecourse of tamoxifen (4OHT) treatment of Sox2FLIP/FLIP CreERT2 ESCs.

C) qRT-PCR of pluripotency factor expression during tamoxifen timecourse. Error bars indicate standard deviation of replicate $\mathrm{qPCR}$ reactions $(\mathrm{n}=3)$. 


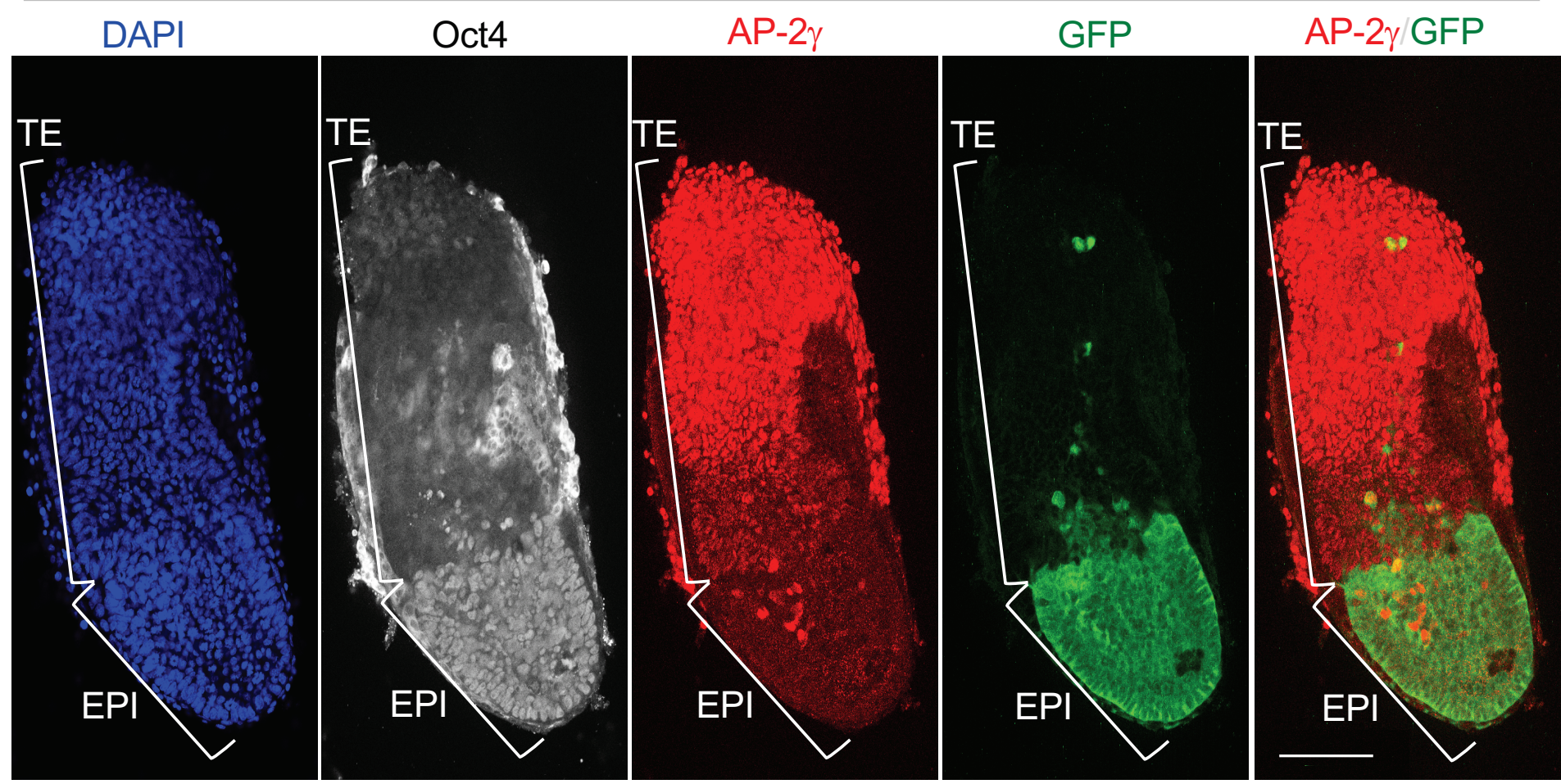

B

-/- Sox2-low blastocyst chimeras

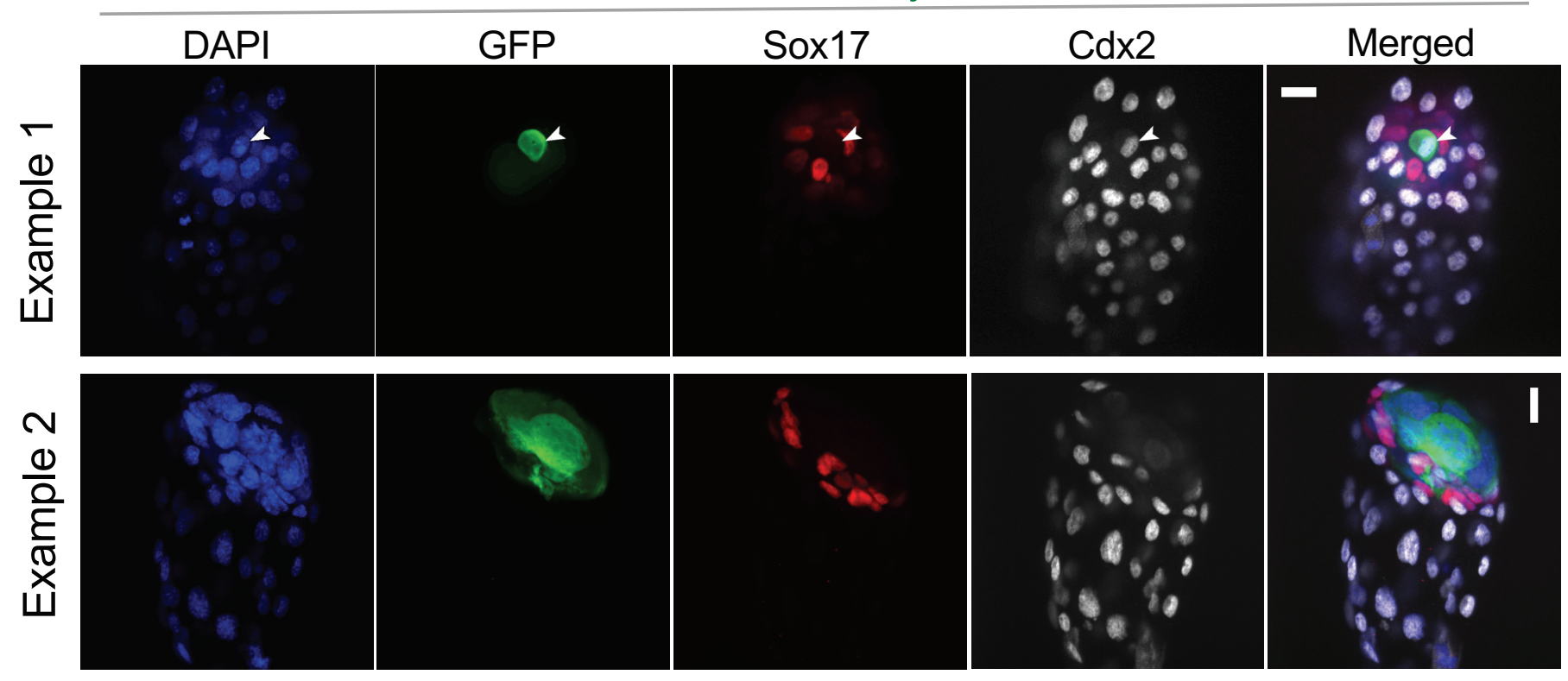

C

-/- Sox2-low blastocyst chimeras

\begin{tabular}{|c|c|}
\hline Epiblast only & 27 \\
\hline Trophectoderm (cdx2 positive) & 1 \\
\hline
\end{tabular}

Figure S3 - Sox2-low iPSCs exhibit increased plasticity in vivo. Related to Figure 5.

A) Single confocal microscopy section of Sox2-- (-/-) Sox2-low iPSCs (GFP) E6.5 chimeric embryos stained with trophoblast (AP-2y) and epiblast (Oct4) markers. Epiblast (EPI) and Trophoblast/trophectoderm (TE) embryo domains are indicated. Scale bars $=100 \mu \mathrm{m}$.

B) Immunofluorescence staining with Cdx2 (trophectoderm) and Sox17 (hypoblast) of cultured embryos after morula injection with Sox2-- (-/-) Sox2-low iPSCs expressing constitutively a GFP transgene. Scale bar $=20 \mu \mathrm{m}$.

C) Table showing compartmental contribution of -/- Sox2-low iPSCs. 
A

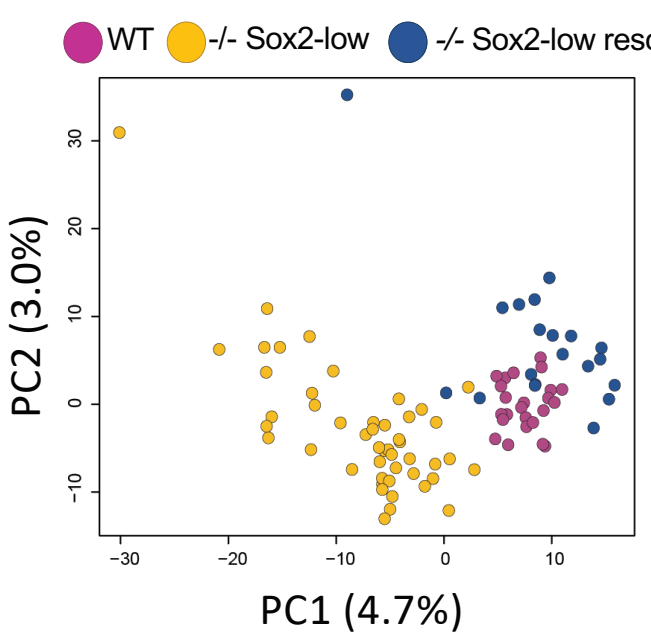

Figure S4- Sox2-low iPSCs do not display gene ontology differences to control cell lines. Related to Figure 6 A) PCA plot computed with the top variable genes for Sox2-- (-/-) Sox2-low and control iPSCs, which include WT and -/- Sox2-low rescued with a constitutive Sox2 transgene (-/- Sox2-low rescue) (FPKM > 1, logCV2 >0.5, $n=1951$ ). The cells are of same genetic background, even the same cell line (parental, knock-out and rescue). They have also been done on same plate for library prep and sequenced in the same lane meaning that the variance is not technical batch effect of the sequencing.

B) Volcano plot and gene ontology enrichment for genes differentially expressed between -/-Sox2-low and control iPSCs (combined WT and -/- Sox2-low rescue iPSCs). log FC indicates comparison between -/- Sox2-low and control iPSCs. Negative value means gene is down regulated in WT and -/- Sox2-low rescue, while positive value indicates gene is upregulated in WT and -/- Sox2-low rescue compared to -/-Sox2-low iPSCs. Significance threshold was defined at $-\log _{10}$ padj $=1.3$. 